\title{
Deep Dive on the Proteome of Human Body Fluids: A Valuable Data Resource for Biomarker Discovery
}

\author{
YAN LI ${ }^{1}$, DAOJIAN XUN ${ }^{1}$, LINGLING LI ${ }^{1}$, BING WANG ${ }^{2}$, JIACHENG LV ${ }^{1}$, HUI LIU $^{2}$, LINGLI ZHU $^{1}$, \\ FAHAN MA ${ }^{1}$, XIUPING CHEN ${ }^{1}$, SHA TIAN $^{1}$ and CHEN DING ${ }^{1,2,3}$ \\ ${ }^{1}$ State Key Laboratory of Genetic Engineering and Collaborative Innovation Center for Genetics and Development, \\ School of Life Sciences, Institute of Biomedical Sciences, \\ Human Phenome Institute, Fudan University, Shanghai, P.R. China; \\ ${ }^{2}$ State Key Laboratory of Cell Differentiation and Regulation, \\ Henan International Joint Laboratory of Pulmonary Fibrosis, \\ Henan Center for Outstanding Overseas Scientists of Pulmonary Fibrosis, \\ College of Life Science, Institute of Biomedical Science, Henan Normal University, Xinxiang, P.R. China; \\ ${ }^{3}$ Academy of Medical Science, Zhengzhou University, Zhengzhou, P.R. China
}

\begin{abstract}
Background/Aim: Body fluids are considered to be a rich source of disease biomarkers. Proteins in many body fluids have potential clinical applications for disease diagnostic and prognostic purposes. The aim of this study was to establish an in-depth multi-body fluid proteome. Materials and Methods: Ten body fluids associated with 8 types of cancers collected from 23 patients involved in 19 common diseases underwent liquid chromatography tandem mass spectrometry (MS) analysis after gel-based protein separation (SDS-PAGE) or peptide-based fractionations. Bioinformatic analysis, including principal component analysis (PCA), consensus clustering, and hierarchical clustering analysis were also performed. The biological function was determined using the Database for Annotation, Visualization and Integrated Discovery (DAVID). Results: We profiled the proteome of ten body fluids, including ascites, bile, cerebrospinal fluid (CSF), hydrothorax, knee joint fluid (KJF), plasma, saliva, serum, tears, and urine. A total of 3,396 nonredundant proteins were identified, of which 304 were shared among ten body fluids, with common functions in focal adhesion and complement/coagulation cascades. A total of $41.5 \%(1,409)$ of the proteins were detected in only one body
\end{abstract}

This article is freely accessible online.

Correspondence to: Professor Chen Ding, State Key Laboratory of Genetic Engineering and Collaborative Innovation Center for Genetics and Development, School of Life Sciences, Institute of Biomedical Sciences, Human Phenome Institute, Fudan University, Shanghai 200433, PR China. Tel: +86 18210311890, Fax: +86 02131246742, e-mail: chend@fudan.edu.cn

Key Words: Body fluids, proteomics, mass spectrometry analysis, biomarkers, gel-based protein separation. fluid and were closely related to their adjacent tissues by function. The functional analysis of the remaining 1,683 proteins showed that similar functions might be shared among different body fluids, which further highlighted the close connection of body fluids in the human body. Conclusion: A deep proteome of multi-body fluids originated from patients diagnosed with 19 common diseases provides a valuable data resource, and might indicate the potential application of body fluids for biomarker discovery.

Human body fluids are considered rich sources of potential disease biomarkers because they are in direct contact with a variety of living tissues, which include plasma/serum, saliva, urine, cerebrospinal fluid (CSF), tear fluid, ascites, bile, hydrothorax and knee joint fluid (KJF). It is widely accepted that human body fluids contain disease-associated proteins that are secreted, shed, and emanated from pathological tissues across the body (1). In disease, highly altered protein expression profiles in body fluids may generate undeniable signatures and unwrapped fingerprints, which may provide insight into disease mechanisms. Therefore, body fluids have potential applicability for the early detection of disease, for the monitoring of its progression, and for therapeutic drug efficacy, especially for their adjacent diseased tissue.

Proteomics is a powerful method for biomedical research. With the significant advances in mass spectrometry (MS) and proteomics technologies $(2,3)$, protein biomarker discovery has become one of the central applications of proteomics. In terms of disease diagnosis and prognosis, compared to tissue biopsy, human body fluids (e.g. blood, urine, tears, or saliva) appear to be more easy and attractive because of several key advantages, including low invasiveness, minimum cost, and the ease of sample collection and processing (4). The 
application of modern proteomic tools in body fluids (5) raised some major issues related to the standardization of sample collection, separation, and processing for qualitative proteomics [such as two-dimensional gel electrophoresis (2DE) (6-8) and multiple liquid chromatography (LC) techniques (9)] or quantitative proteomics [such as 2D difference gel electrophoresis (10), isotope-coded affinity tag (11), stable isotope labeling by amino acids in cell culture $(12,13)$ and isobaric tags for relative and absolute quantification (14-17)]. The ion intensity-based label-free quantitative approach has gradually gained more popularity and provides an alternative powerful tool to resolve and identify thousands of proteins from a complex biological sample $(18,19)$.

Body fluid proteomes have been investigated for more than 10 years, beginning with the Human Plasma Proteome Project in 2002 (20). The emergence of high-accuracy and highresolution mass spectrometers in 2006 led to large-scale analyses of tear-duct fluid (21), urine (22), and seminal plasma (23) from Mann's group, which were considered the first high-accuracy proteome maps of human body fluids. With the rapid development of MS technology, the large-scale MS-based draft of the human proteome was presented in 2014 (24), including the proteomes of human tissues, cell lines, and body fluids. Among them, the proteomic datasets from eight body fluids (urine, seminal plasma, blood, vitreous humor, CSF, synovial fluid, saliva, and milk) were assembled to contribute to the whole proteome map of humans (24).

Urine with less complexity than serum and relatively high thermodynamic stability is a promising study medium for discovery of novel biomarkers in many human diseases, such as bladder and prostate cancer, and renal diseases (25-27). CSF is secreted from several different central nervous system (CNS) structures, and the change in the protein composition of CSF is considered a sensitive indicator for the analysis of neurodegenerative or other CNS disorders $(28,29)$. As for saliva, the related salivary protein markers (such as glycoproteins) (30) have been well demonstrated to be important for the diagnosis of Sjogren's syndrome (SS), an autoimmune disease characterized by xerostomia (dry mouth) and xerophthalmia (dry eyes). Tear fluid analysis is also a noninvasive approach in early diagnosis and study of pathogenesis of eye-related diseases such as dry eye syndrome.

In this study, we collected 10 body fluids from 23 patients diagnosed with 19 common diseases, obtained 157 MS raw files, and conducted a proteomic comparison using highresolution MS. All of the proteomic data from ascites, bile, CSF, hydrothorax, KJF, plasma, saliva, serum, tears and urine were obtained using similar approaches and bioinformatics pipelines. For each body fluid, relative protein abundance was estimated using intensity-based algorithm quantitation (iBAQ) methods (31). A deep analysis was performed regarding the protein composition of ten body fluids, and the proteins were extensively characterized to provide functional annotation of the body fluid proteomes, novel insights into the relationships among these ten body fluids, and greater research utility for multiple diseases.

\section{Materials and Methods}

Body fluid samples. Prior to enrollment, all volunteers were provided with a verbal explanation of the study and signed a document of informed consent. The study was approved by the Institutional Research Ethics Committee of Zhongshan Hospital (B2019-200R). For the complex proteome database of human body fluids, we collected 10 body fluids (ascites, bile, CSF, hydrothorax, KJF, plasma, saliva, serum, tears and urine) from 23 patients diagnosed with 19 common diseases (10 females and 13 males, mean age of 52 years). The characteristics of the 23 patients are shown in Table I.

Sample preparation. All the samples were centrifuged at $12,000 \times \mathrm{g}$, at $4^{\circ} \mathrm{C}$ for $10 \mathrm{~min}$ to remove the cells and any debris. The supernatants were collected, and the protein concentration was determined using the Bradford assay (TaKaRa, Beijing, PR China). The samples were stored at $-80^{\circ} \mathrm{C}$ until further use.

Protein trypsin digestion and one-dimensional reversed-phase liquid chromatography (RPLC). Two strategies were adopted for further analysis. In the first strategy, ascites, bile, CSF, hydrothorax, KJF, plasma, serum, and urine samples were depleted of high-abundance proteins, fractioned on SDS-PAGE and in-gel tryptic digestion. Depletion of human serum albumin (HSA), albumin, IgG, IgA, IgM, $\operatorname{IgD}, \operatorname{IgE} \mathrm{kB}$ and $\lambda$ light chains, $\alpha$-1-acidic glycoprotein, $\alpha-1$ antitrypsin, $\alpha$-2-macroglobulin, apolipoprotein A1, fibrin, binding globin and transferrin from serum and plasma was carried out using High-Select ${ }^{\mathrm{TM}}$ Top14 Abundant Protein Depletion Mini Spin Columns (Thermo Fisher Scientific, Rockford, IL, USA) as per the manufacturer's instructions. The depleted proteins were resolved on a $4 \%-20 \%$ gradient SDS-PAGE gel and the gel was stained using colloidal Coomassie blue. The dark-colored gel slices were excised and destained using $50 \mathrm{mM}$ ammonium bicarbonate (ABC) in $40 \%$ methanol and then sequentially vibrated in $75 \%$ acetonitrile $(\mathrm{ACN})$, HPLC/MS grade water, and $50 \mathrm{mM} \mathrm{ABC}$. The sample was then subjected to reduction using $5 \mathrm{mM}$ dithiothreitol (DTT) $\left(60^{\circ} \mathrm{C}\right.$ for 45 $\mathrm{min}$ ) followed by alkylation using $20 \mathrm{mM}$ iodoacetamide $(10 \mathrm{~min}$ at room temperature). In-gel digestion with trypsin was carried out at $37^{\circ} \mathrm{C}$ for $12-16 \mathrm{~h}$. Peptides were extracted from gel pieces sequentially using $100 \%$ ACN, $0.1 \%$ formic acid, and $100 \%$ ACN. The tryptic peptides were desalted by a home-made reverse-phase $\mathrm{C} 18$ column in a pipet tip, and eluted with acetonitrile $(50 \%)$ and formic acid $(0.1 \%)$. The peptide elution was dried in a vacuum concentrator (Concentrator plus, Eppendorf, Hamburg, Germany), and then analyzed by liquid chromatography tandem mass spectrometry (LC-MS/MS).

Alternatively, another strategy used for saliva and tear fluid was to perform direct in-solution digestion with trypsin; the resulting peptides were then fractionated by a home-made reverse-phase C18 column in a pipet tip. Peptides were eluted and separated into nine fractions using a stepwise gradient of increasing acetonitrile $(6 \%$, $9 \%, 12 \%, 15 \%, 18 \%, 21 \%, 25 \%, 30 \%$, and $35 \%$ ) at $\mathrm{pH} 10$. The nine fractions were combined to three fractions $(6 \%+15 \%+25 \%$; $9 \%+18 \%+30 \% ; 12 \%+21 \%+35 \%)$. The peptide elution was dried in 
Table I. Data of patients used for human body fluids proteomic analysis.

\begin{tabular}{|c|c|c|c|c|}
\hline Sample ID & Body fluids & Gender & Age (years) & Diagnosis \\
\hline Case 1 & Ascites & Male & 41 & SBS \\
\hline Case 2 & Ascites & Female & 43 & Pancreatic lesions \\
\hline Case 3 & Ascites & Male & 44 & Liver lesions \\
\hline Case 4 & Ascites & Female & 40 & Choledochal cyst \\
\hline Case 5 & Bile & Male & 70 & Duodenal papillary adenoma with high intraepithelial neoplasia \\
\hline Case 6 & Bile & Male & 80 & Acute cholangitis \\
\hline Case 7 & CSF & Female & 59 & SAH \\
\hline Case 8 & CSF & Female & 31 & Chair deformity with syringomyelia \\
\hline Case 9 & CSF & Female & 62 & The right cerebellar hemangioblastoma \\
\hline Case 10 & Hydrothorax & Male & 48 & Pleural effusion \\
\hline Case 11 & Hydrothorax & Female & 73 & Hepatitis cirrhosis \\
\hline Case 12 & Hydrothorax & Male & 82 & Gastrointestinal hemorrhage \\
\hline Case 13 & KJF & Female & 29 & SLE \\
\hline Case 14 & Plasma & Male & 37 & Intracranial aneurysm \\
\hline Case 15 & Plasma & Female & 64 & Cholecystolithiasis with chronic cholecystitis \\
\hline Case 16 & Plasma & Male & 66 & Chest distress \\
\hline Case 17 & Saliva & Female & 28 & Normal \\
\hline Case 18 & Serum & Male & 45 & Pulmonary fibrosis \\
\hline Case 19 & Serum & Male & 55 & Pulmonary fibrosis \\
\hline Case 20 & Tear & Female & 28 & Normal \\
\hline Case 21 & Urine & Male & 52 & Hepatitis B cirrhosis \\
\hline Case 22 & Urine & Male & 62 & Abdominal aortic aneurysm \\
\hline Case 23 & Urine & Male & 64 & Liver lesions \\
\hline
\end{tabular}

KJF: Knee joint fluid; SBS: short-bowel syndrome; SAH: subarachnoid hemorrhage; SLE: systemic lupus erythematosus.

a vacuum concentrator (Concentrator plus, Eppendorf), and then analyzed by LC-MS/MS.

LC-MS/MS analysis. Peptides from body fluids were detected by Orbitrap Fusion Lumos (Thermo Fisher Scientific). Orbitrap Fusion Lumos LC-MS/MS analyses were performed on an Easy-nLC 1000 liquid chromatography system (Thermo Fisher Scientific) coupled to an Orbitrap Fusion Lumos via a nano electrospray ion source (Thermo Fisher Scientific). Fractions from the first-dimension RPLC were dissolved with a loading buffer (5\% methanol and $0.2 \%$ formic acid) and loaded onto a $360 \mu \mathrm{m} \mathrm{ID} \times 2 \mathrm{~cm}, \mathrm{C} 18$ trap column at a maximum pressure of 280 bar with $12 \mu \mathrm{l}$ of solvent A $(0.1 \%$ formic acid in water). Peptides were separated on a $150 \mu \mathrm{m}$ ID $\times 14 \mathrm{~cm}$ column $(\mathrm{C} 18,1.9 \mu \mathrm{m}, 120 \AA$, Dr. Maisch $\mathrm{GmbH}$, Germany) over a 75 -min gradient (Solvent A: $0.1 \%$ formic acid in water; Solvent B: $0.1 \%$ formic acid in $80 \% \mathrm{ACN}$ ) at a constant flow rate of $600 \mathrm{Nl} / \mathrm{min}$. The 75 -min gradient was set as following: $4 \%-15 \% \mathrm{~B}$ in $10 \mathrm{~min} ; 15 \%-30 \% \mathrm{~B}$ in $50 \mathrm{~min} ; 30 \%-50 \% \mathrm{~B}$ in 9 $\min ; 50 \%-100 \% \mathrm{~B}$ in $1 \mathrm{~min} ; 100 \% \mathrm{~B}$ for $5 \mathrm{~min}$. The eluted peptides were ionized under $2 \mathrm{kV}$ and introduced into the mass spectrometer. MS was operated under a data-dependent acquisition mode. For the MS1 Spectra full scan, ions with $\mathrm{m} / \mathrm{z}$ ranging from 300 to 1,400 were acquired by Orbitrap mass analyzer at a high resolution of 60,000 . The automatic gain control (AGC) target value was set at $4.0 \mathrm{E}+05$. The maximal ion injection time was 50 ms. MS2 Spectra acquisition was performed in quadrupole isolation mode with an isolation window of $1.6 \mathrm{~m} / \mathrm{z}$. Precursor ions were selected and fragmented with higher energy collision dissociation (HCD) with a normalized collision energy of $30 \%$. Fragment ions were analyzed using an ion trap mass analyzer with an AGC target value of $5 \mathrm{E}+04$ at a high resolution of 15,000 , with a maximal ion injection time of $22 \mathrm{~ms}$. Peptides that triggered MS/MS scans were dynamically excluded from further MS/MS scans for $18 \mathrm{~s}$. All data were acquired using the Xcalibur software (Thermo Fisher Scientific).

Peptide identification and label-free-based protein quantification. MS raw files were searched against the NCBI human Refseq protein database (released on 04-07-2013; 32,015 entries) with MaxQuant software, using the integrated Andromeda search engine with the false discovery rate (FDR) $<1 \%$ at peptide and protein levels. The mass tolerances were $20 \mathrm{ppm}$ for the precursor and $0.5 \mathrm{Da}$ for productions collected by Fusion Lumos. The proteolytic cleavage sites were $\mathrm{K}$ and $\mathrm{R}$. Up to two missed cleavages were allowed. The database search considered cysteine carbamidomethylation as a fixed modification and $\mathrm{N}$-acetylation and oxidation of methionine as variable modifications. All identified peptides were quantified in MaxQuant derived from their MS1 intensity. Peptide FDR was adjusted to $1 \%$. For protein quantification, we used intensity-based label-free quantification, known as the iBAQ algorithm, which divided the protein abundance (derived from identified peptides' intensities) by the number of theoretically observable peptides. A match between runs (MBR) (32) was enabled to transfer the identification between separate LC-MS/MS runs based on their accurate mass and retention time after retention time alignment. The $\mathrm{z}$-score was calculated using the equation $\mathrm{z}=(\mathrm{x}-\mu) / \sigma$, where $\mu$ stands for the mean of the samples' iBAQ and $\sigma$ stands for the standard deviation of the samples. 
Bioinformatics and statistical analysis for MS data. Principal component analysis (PCA) of 10 body fluids was performed using the FactoMineR package (version 2.4) (33). The protein expression matrix of 10 body fluids was used to identify the proteomic clusters using the consensus clustering method implemented in the $\mathrm{R}$ package ConsensusClusterPlus v.3.8 (34). Consensus clustering analysis of the proteomic profiles identified four clusters of 10 body fluids based on the 1,500 most variable proteins (proteins with the top 44\% standard deviations). The cluster analysis was performed using $\mathrm{k}$-means, with the following setting: $\operatorname{maxK}=8$, reps $=10,000$, pItem $=0.8$, pFeature $=1$, clusterAlg="hc", distance="pearson" for the clustering runs. Preferred cluster results were selected by considering the profiles of the consensus cumulative distribution function (CDF) and delta area under the CDF curve for clustering solutions between 2 and 8 clusters. The rank survey profiles of the consensus CDF and the delta area under the CDF curve indicated a four-cluster solution for 10 body fluids using the proteomic data. Hierarchical clustering was performed using the pheatmap (Pretty Heatmaps) function in the $\mathrm{R}$ package (pheatmap, version 1.0.8). The Gene Ontology (GO) biological process (GO-BP), the GO cellular component (GO-CC) and GO molecular function (GO-MF) terms, and the Kyoto Encyclopedia of Genes and Genomes (KEGG) pathways that were enriched in the sets of enriched genes were determined using the Database for Annotation, Visualization and Integrated Discovery (35) Bioinformatics Resource v 6.8. The values of $p<0.05$ and FDR $<0.05$ (adjusted by Bonferroni and Benjamini) were considered to determine the statistically significant results of GO-BP, GO-CC, GO-MF and KEGG enrichment. Pearson's correlation analysis was used to calculate the correlation coefficients (r) among 10 body fluids.

Data availability. The accession number for the MS proteomics data reported in this paper is iProX repository (www.iprox.org) (36): Project ID IPX0002854000 and ProteomeXchange ID PXD024255.

\section{Results}

Proteomic analysis of 10 body fluids. In this study, we conducted proteome profiling of 10 human body fluids (ascites, bile, CSF, hydrothorax, KJF, plasma, saliva, serum, tears, and urine) through gel-based protein separation (SDS-PAGE) or peptide-based fractionations and then LC-MS/MS identification with Orbitrap Fusion Lumos (Figure 1A). For gel-based protein separation, we obtained the gel-band fractions of body fluids as follows: ascites (31 fractions), bile (12 fractions), CSF (17 fractions), hydrothorax (19 fractions), KJF (17 fractions), plasma (28 fractions), serum (18 fractions), and urine (9 fractions). For peptide-based fractionations, we combined the nine fractions into three fractions: F1 $(6 \%+15 \%+25 \%), \mathrm{F} 2$ $(9 \%+18 \%+30 \%)$, and F3 $(12 \%+21 \%+35 \%)$. Overall 157 raw files of body fluids were obtained. The high-resolution tandem mass spectra were searched against the human RefSeq protein database and then imported into the MaxQuant software to estimate the false discovery rates for the proteins. Matches between runs were adopted for fractions within one body fluid. The FDRs for protein identifications were controlled to less than $1 \%$. A total of 3,396 proteins were identified from the MS raw data. For samples such as bile, ascites, and plasma that were separated with SDS-PAGE, the number of protein identifications was higher than the proteins identified in saliva and tears. A combined list of 2,109, 1,892, and 1,277 proteins were identified in the bile, ascites, and plasma, respectively. A total of 827 and 459 proteins were identified in the untreated saliva and tear fluid, respectively (Figure 1B).

Quantification of the body fluids proteome. Using the intensity-based absolute quantification (iBAQ) algorithm, we estimated the overall distribution of the protein abundance in 10 body fluids based on the original quantitative information detected by label-free quantification. The proteome quantification results of 10 body fluid experiments were relatively comparable, as their medians were on the same level (Figure 1C). In 10 body fluid proteomes, the iBAQ values varied by approximately 3 to 12 orders of magnitude, indicating a highly dynamic range (Figure 1D). The top 10 ranked proteins with high or low abundance of 10 body fluids are listed as shown in Figure 1E. We found that plasma and serum were enriched by plasma lipoproteins such as APOA1, APOA2, APOA3, and APOA4, and complement activation regulators such as $\mathrm{C} 3$ and $\mathrm{C} 4 \mathrm{~B}$. In addition, the proteins involved in complement and coagulation cascades such as FGA, SERPINA1, SERPINC1, and SERPING1, were also overrepresented in other body fluids such as ascites, bile, and hydrothorax. The low abundance proteins of 10 body fluids were almost involved in immune system and immune response, e.g. UBE2V1, UBE2K, PTPN11, TNFSF13B, and VCAM1. For all 3,396 proteins identified by LC-MS/MS analysis, approximately $58.5 \%(1,987)$ were identified in at least two body fluids, of which $9.0 \%$ (304) were commonly identified in 10 body fluids, and the remaining $41.5 \%(1,409)$ of "specific" proteins were identified in only one body fluid (Figure 1F).

Figure 1. Summary of the proteomic analysis of body fluids. (A) Schematic illustration of the experimental workflow. Two strategies for body fluids were adopted: in one, the proteins were separated on SDSPAGE and in-gel digested with trypsin; in the other, the proteins were digested in-solution. The peptides were then analyzed by liquid chromatography tandem mass spectrometry (LC-MS/MS) using an Orbitrap Fusion Lumos. (B) The bar plot indicates the number of proteins detected in ten body fluids. (C) The boxplot illustrates the protein abundance of ten body fluids. (D) Dynamic range of ten body fluids, based on protein abundance [Log 10 (iBAQ)]. (E) List of proteins with high abundance or low abundance of each body fluid. $(F)$ Pie chart showing the protein identification among ten body fluids. (G) The KEGG pathways of all identified proteins in ten body fluids. KJF: Knee-joint fluid; CSF, cerebrospinal fluid; KEGG: Kyoto Encyclopedia of Genes and Genomes; TCA: tricarboxylic acid cycle. 
A

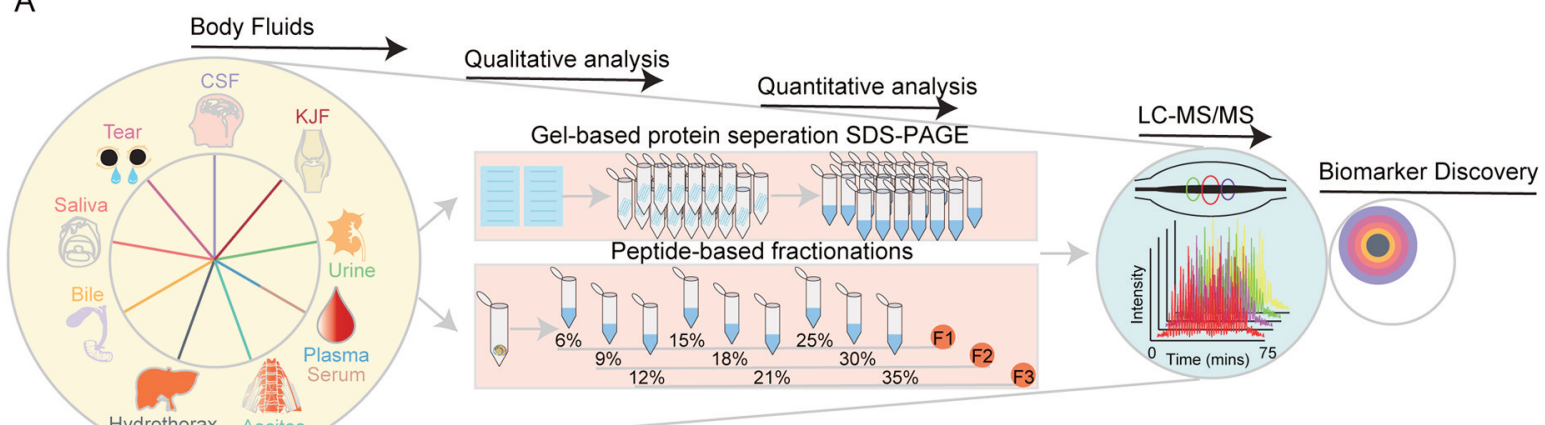

B

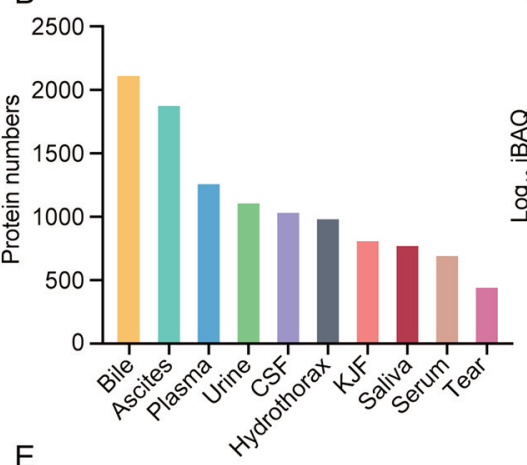

E

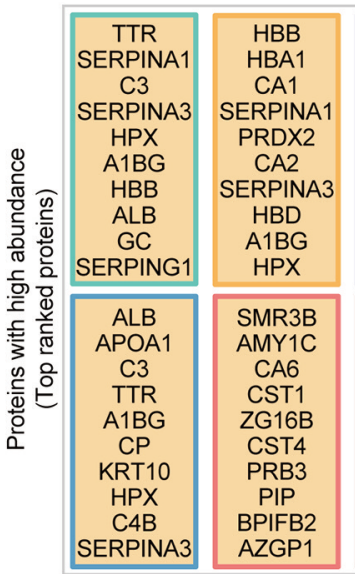
HBB
HBA2
TTR
PTGDS
CA1
HBD
SERPINA3
APOA1
APOE
PRDX2

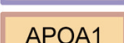

APOA $A 1 B G$
APOA2 C3 APOA4 HPX SERPINC APOC3 SERPINA3
C

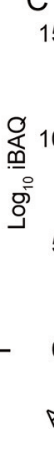

D
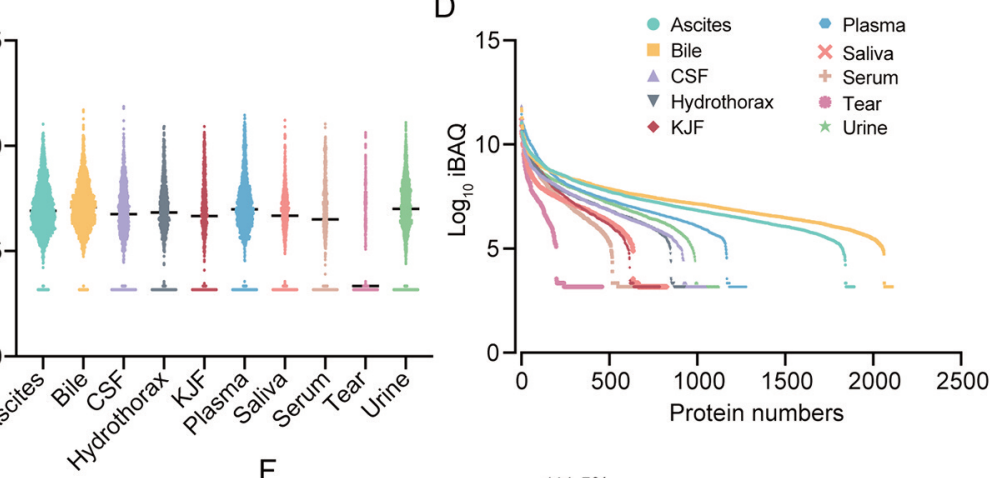

$\mathrm{F}$

\begin{tabular}{|c|c|}
\hline APOA1 & TTR \\
TTR & APOA1 \\
ALB & APERINA3 \\
APOA4 & SEROA4 \\
APOA2 & APBB \\
AZGP1 & HBB \\
KRT1 & ALB \\
APOE & HBA1 \\
FGA & ABG \\
CRP & HPX \\
\hline LC3 & C3 \\
\hline LCN1 & RBP4 \\
PIP & AMBP \\
PROL1 & PTGDS \\
SMR3B & TTR \\
PRR4 & CST3 \\
LACRT & LCN2 \\
LYZ & GM2A \\
AZGP1 & LCN1 \\
LTF & REG1B \\
SCGB2A1 & R2M \\
\hline
\end{tabular}

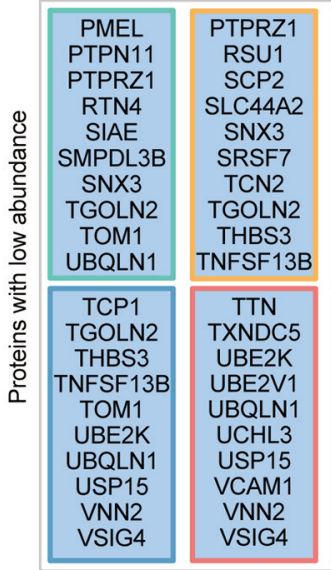

\begin{tabular}{|l|}
\hline STOM \\
SYNCRIP \\
TCP1 \\
THBS3 \\
TOM1 \\
TPM2 \\
TUBA4A \\
UBE2K \\
UCHL3 \\
VNN2 \\
\hline TPM2 \\
TXNDC5 \\
UBE2K \\
UBE2V1 \\
UBQLN1 \\
UCHL3 \\
USP15 \\
VNN2 \\
VSIG4 \\
WARS \\
\hline
\end{tabular}

\begin{tabular}{|c|c|} 
THBS3 & TUBA4A \\
TOM1 & TXN \\
TPM2 & TXNDC5 \\
TTNN & UBE2K \\
TUBA4A & UBE2V1 \\
UBE2K & UBQLN1 \\
UBQLN1 & UCHL3 \\
USP15 & USP15 \\
VNN2 & VNN2 \\
WARS & WARS \\
\hline \hline TXNDC5 & TCN2 \\
UBE2K & TCP1 \\
UBE2V1 & THBS3 \\
UBQLN1 & TPM2 \\
UCHL3 & TUBA4A \\
USP15 & UBE2K \\
VCAM1 & UBQLN1 \\
VNN2 & UCHL3 \\
VSIG4 & USP15 \\
WARS & WARS \\
\hline
\end{tabular}

$$
\begin{gathered}
(9.0 \%, \\
\mathrm{N}=304)
\end{gathered}
$$

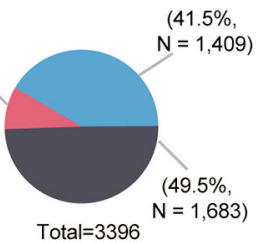

G

KEGG pathway overrepresented hsa01130:Biosynthesis of antibiotics hsa04145: Phagosome hsa01200:Carbon metabolism hsa04142:Lysosome hsa04610:Complement and coagulation cascades hsa05146: Amoebiasis hsa04512:ECM-receptor interaction hsa04974:Protein digestion and absorption hsa01230:Biosynthesis of amino acids hsa00010: Glycolysis / Gluconeogenesis hsa03050:Proteasome hsa05150: Staphylococcus aureus infection hsa05130:Pathogenic Escherichia coli infection hsa00480:Glutathione metabolism hsa00071: Fatty acid degradation hsa05144:Malaria hsa00280:Valine, leucine and isoleucine degradation hsa05020:Prion diseases hsa00620:Pyruvate metabolism hsa00350:Tyrosine metabolism hsa00030:Pentose phosphate pathway hsa00500:Starch and sucrose metabolism hsa00051:Fructose and mannose metabolism hsa00020: Citrate cycle (TCA cycle) -

$$
\begin{gathered}
50 \quad 100150 \\
\text { Count }
\end{gathered}
$$


The overall proteomic characterization of body fluids. Gene Ontology (GO) annotation and Kyoto Encyclopedia of Genes and Genomes (KEGG) pathway analysis of the relative abundances of all identified proteins across 10 body fluids were performed. A total of 3,396 proteins were clustered into 262, 300, and 797 terms in the GO cellular component (GO$\mathrm{CC})$, molecular function (GO-MF), and biological process (GO-BP) categories, respectively. Among them, 125 (47.4\%), $96(32.0 \%)$, and 205 terms $(25.7 \%)$ exhibited significance $(p<0.001)$ as the overrepresented terms in GO-CC, GO-MF, and GO-BP, respectively. The overrepresented terms were filtered with a count of more than 20 , enrichment greater than 2-fold, and FDR less than 0.001. In the GO-CC category, GO terms related to extracellular proteins such as extracellular exosome (1,695 proteins found), extracellular space (611), and extracellular matrix (202) were overrepresented. GO terms related to plasma membrane proteins $(105, \mathrm{FDR}=5.20 \mathrm{E}-12)$, blood microparticle $(101, \mathrm{FDR}=2.22 \mathrm{E}-37)$, and lysosome proteins $(96, \mathrm{FDR}=3.82 \mathrm{E}-16)$ were overrepresented. In the GO-MF category, most proteins (156, FDR=1.08E-38) were clustered into cadherin binding involved in cell-cell adhesion, as expected. As for the GO-BP category, the top ranked terms were platelet degranulation $(81, \mathrm{FDR}=3.10 \mathrm{E}-35)$, cell adhesion (206, FDR=3.10E-35), proteolysis $(189, \mathrm{FDR}=2.28 \mathrm{E}-21)$, cellcell adhesion $(140, \mathrm{FDR}=4.04 \mathrm{E}-31)$, and extracellular matrix organization $(105, \mathrm{FDR}=1.86 \mathrm{E}-24)$. The KEGG pathway analysis identified 48 overrepresented terms, which were clustered into 2 major systems: immune system and metabolism system. The immune system included biosynthesis of antibiotics (112, FDR=1.03E-15), phagosome (89, $\mathrm{FDR}=3.08 \mathrm{E}-16)$, complement and coagulation cascades $(62$, $\mathrm{FDR}=5.57 \mathrm{E}-26)$, and extracellular matrix (ECM)-receptor interaction (54, FDR=7.51E-11). The metabolism system was composed of carbon metabolism (69, FDR=1.92E-13), glycolysis/gluconeogenesis $(42, \mathrm{FDR}=1.35 \mathrm{E}-8)$, glutathione metabolism $(28, \mathrm{FDR}=1.83 \mathrm{E}-4)$, fatty acid degradation $(28$, FDR=1.62E-6), pyruvate metabolism $(24, \mathrm{FDR}=1.38 \mathrm{E}-4)$, and tyrosine metabolism $(22, \mathrm{FDR}=1.36 \mathrm{E}-4)$ (Figure $1 \mathrm{G})$. These findings presented a comprehensive molecular feature of multi-body fluids, pinpointing to the immune and metabolic systems as the main axes of biological function.

Consensus clustering analysis of 10 body fluids based on proteome. Consensus clustering analysis of the proteomic profiles identified four clusters of 10 body fluids based on the 1,500 most variable proteins (proteins with the top $44 \%$ standard deviations) (Figure 2). Ascites, bile, and CSF were grouped into cluster C-I, hydrothorax, KJF, plasma and serum were grouped into cluster C-II, while saliva and tears were grouped into cluster C-III; urine was solely grouped into cluster C-IV (Figure 3A and Table II). The consensus clustering result showed the similarity of biological function in each body fluid cluster. Next, we performed a functional enrichment analysis according to KEGG pathway annotations, and determined the dominant bioprocesses of each cluster (Figure 3B and 3C). The C-I cluster was associated with metabolic bioprocesses, including carbon metabolism ( $p=1.23 \mathrm{E}-19)$ (e.g. GPI, ENO1, G6PD, etc.), glycolysis/ gluconeogenesis ( $p=6.47 \mathrm{E}-13)$ (e.g . ADH1C, ADH1A, PGK1, etc.), fatty acid degradation ( $p=6.20 \mathrm{E}-9)$ (e.g. ACAA1, ACAT1, HADH, etc.), glyoxylate and dicarboxylate metabolism ( $p=3.06 \mathrm{E}-7)$ (e.g. MDH1, GRHPR, PGP, etc.). The C-II cluster, containing hydrothorax, $\mathrm{KJF}$, plasma, and serum, was associated with metabolismrelated oxidative phosphorylation ( $p=1.03 \mathrm{E}-5)(e . g$. NDUFA8, UQCRB, COX7A2, etc.), as well as immune bioprocesses, including complement and coagulation cascades $(p=1.24 \mathrm{E}-53)$ (e.g. C1QA, C1R, C1S, etc.), ECM-receptor interaction $(p=4.27 \mathrm{E}-6)(e . g$. THBS3, ITGA2, CD36, etc.), and focal adhesion ( $p=3.31 \mathrm{E}-5)$ (e.g. COL4A1, VWF, EGFR, etc.). The C-III cluster was characterized with salivary secretion ( $p=3.84 \mathrm{E}-7)$ (e.g. CST1, CST2, DMBT1, etc.), sphingolipid signaling pathway $(p=2.25 \mathrm{E}-2)(e . g$. PPP2R2B, SPTLC2, SGPP1, etc.), fatty acid biosynthesis ( $p=2.28 \mathrm{E}-2)$ (e.g. ACSL1, FASN, ACSBG2, etc.), and apoptosis ( $p=2.65 \mathrm{E}-2)$ (e.g. AKT3, FADD, BID, etc.). While in $\mathrm{C}-\mathrm{IV}$, the urinary proteome was enriched by lysosome related proteins (such as ATP6V0C, AGA, and CSTC), cell adhesion molecules (CAMs) (such as CD276, CDH3, and HLA-DRB1), and ECM receptors (such as LAMA5, LAMB2, and COL6A3) (Figure 3B and 3C).

The specific protein components and functional features of 10 body fluids. Correlation coefficients (Pearson's correlation) among 10 body fluids showed a broad range of 0 to 0.87 (Figure 4A). Among them, ascites showed higher correlation with hydrothorax, KJF, and plasma $(r=0.63,0.87$ and 0.64 , respectively), of which the latter three body fluids showed higher correlation with each other $(\mathrm{r}=0.78,0.73$ and 0.75 , respectively). Serum highly correlated with hydrothorax, KJF, and plasma ( $\mathrm{r}=0.74,0.64$ and 0.83 , respectively). On the contrary, we found that bile showed very low correlation with hydrothorax $(\mathrm{r}=0.06)$ and urine $(\mathrm{r}=0.03)$. The overlapping protein numbers of ascites, hydrothorax, KJF, and plasma are also respectively shown in Figure 4B. PCA showed that the protein components of 10 body fluids were distinctive (Figure 4C). Furthermore, we performed a comparison of these body fluid proteome datasets. For all 3,396 proteins identified by LCMS/MS analysis, approximately $58.5 \%(1,987)$ were identified in at least two body fluids and the remaining $41.5 \%(1,409)$ of "specific" proteins were identified in one body fluid, providing clues that the "specific" proteins can be used for biomarker discovery. In detail, the "specific" proteins identified in ascites, bile, CSF, hydrothorax, KJF, plasma, saliva, serum, tears, and urine were separately 279, 482, 92, 43, 25, 108, 117, 26, 71, and 166, respectively (Figure 4D). The GO and KEGG analysis of the body fluid-specific proteins revealed that the proteins were closely related to the tissues and their adjacent tissues by 
consensus matrix legend

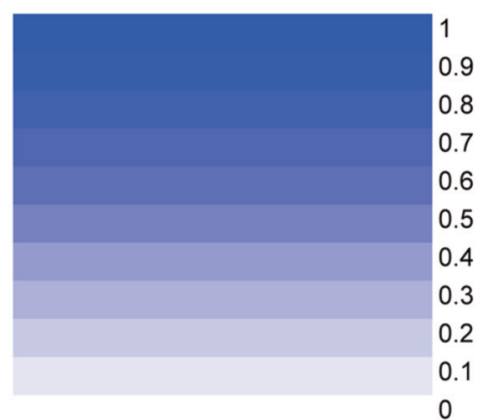

consensus matrix $\mathrm{k}=4$
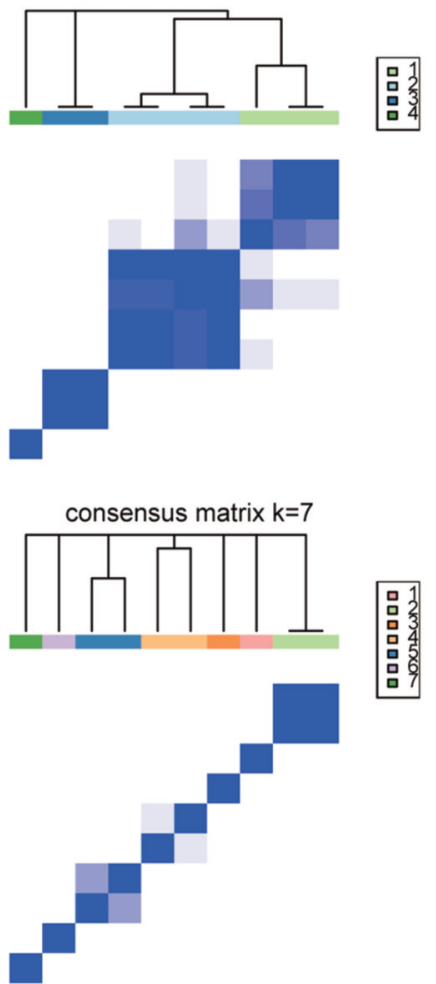

consensus CDF
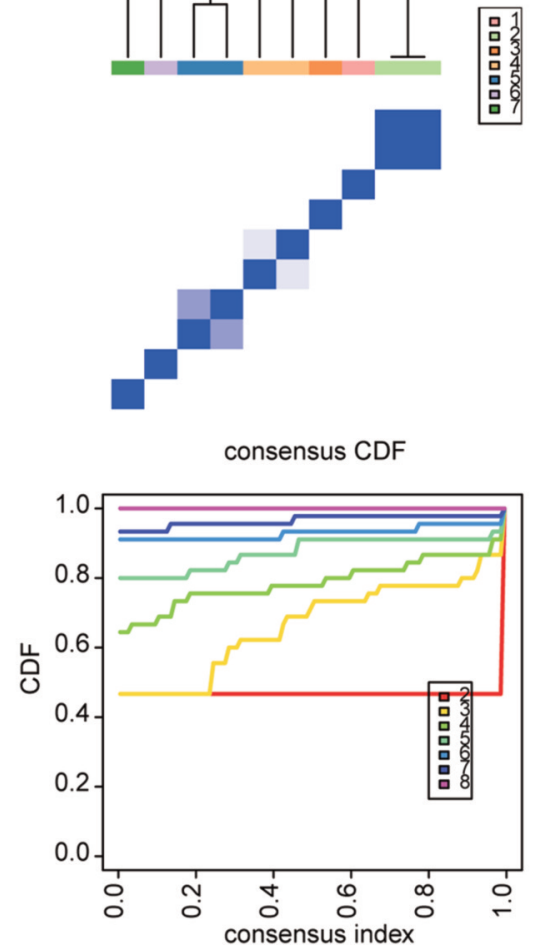

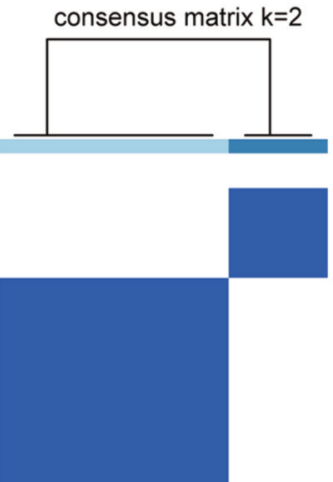

圆

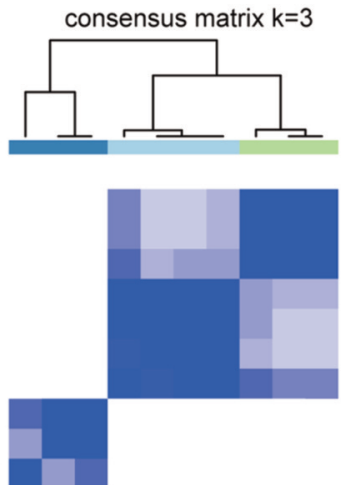

\begin{tabular}{|l|}
\hline 吕 \\
3
\end{tabular}

consensus matrix $\mathrm{k}=5$

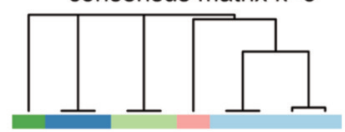

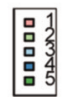

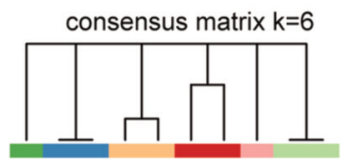

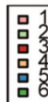
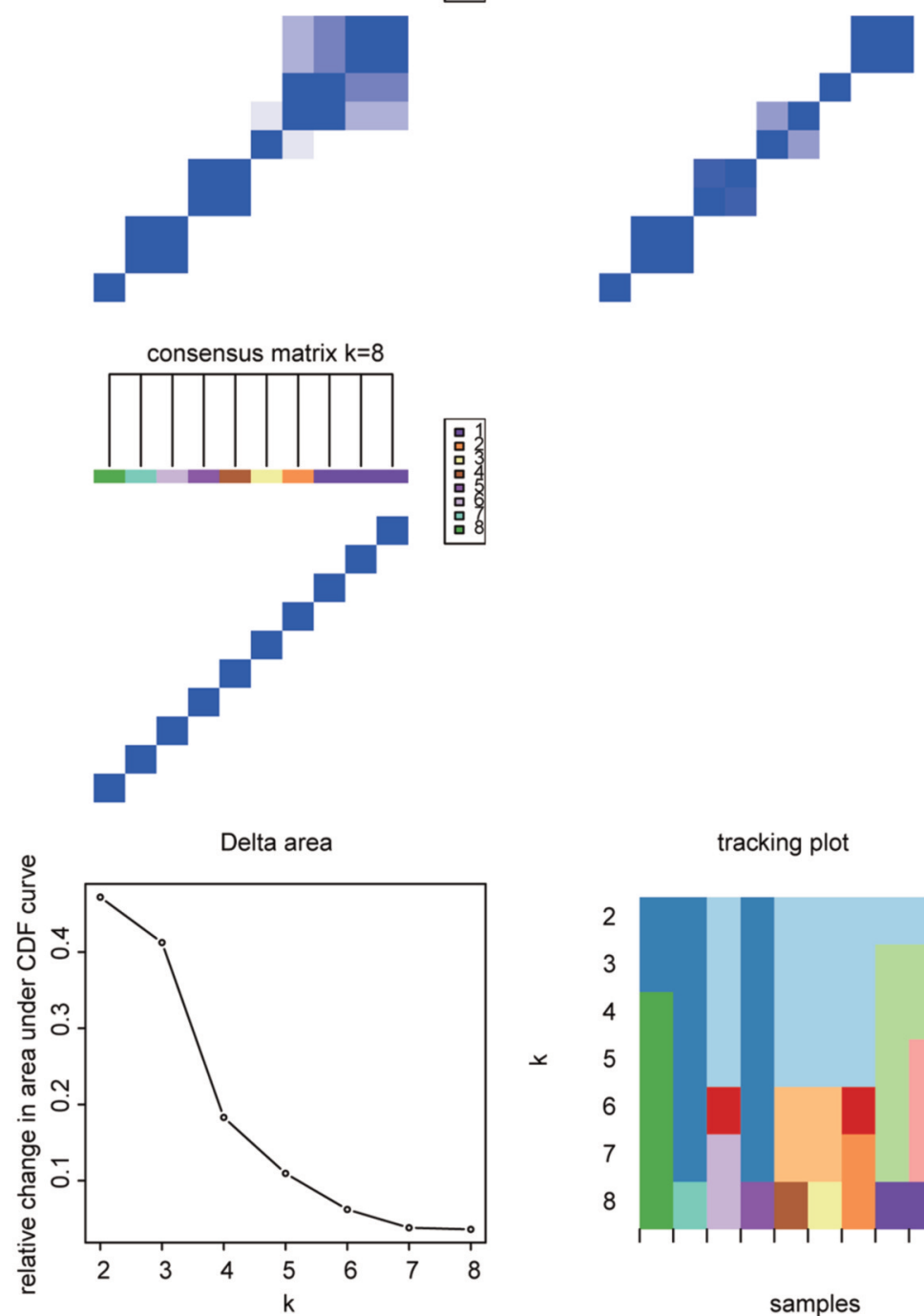

tracking plot

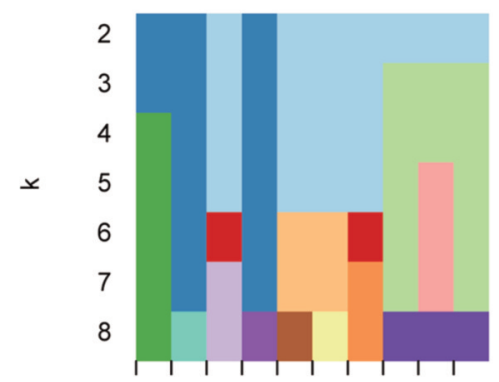

samples

Figure 2. The consensus clustering analysis of ten body fluids. The consensus clustering analysis of ten body fluids identified four clusters. $k$ was tested from 2 to 8. Consensus matrices, as well as the consensus cumulative distribution function (CDF) plot, delta area (change in CDF area) plot, and tracking plot are shown. 
A

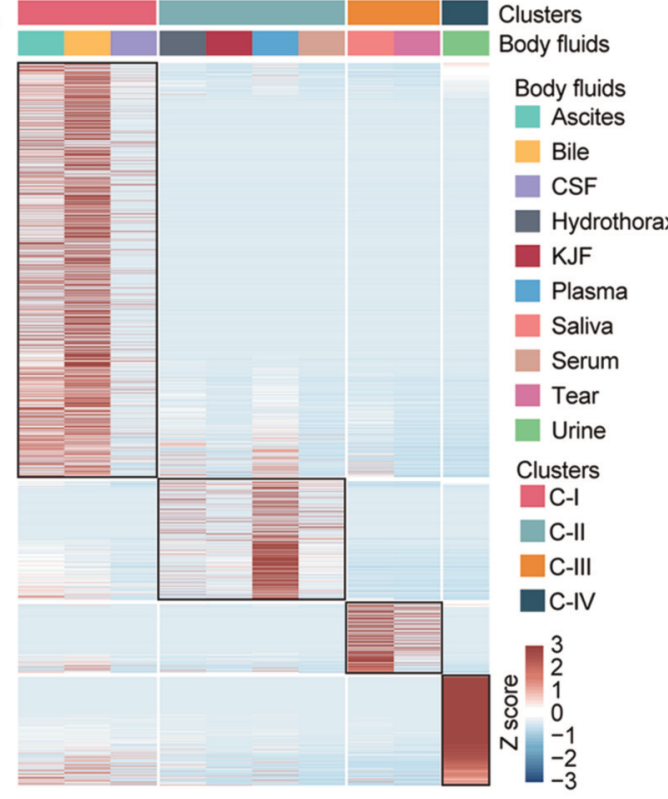

C

Glycolysis/Gluconeogenesis \& Fatty acid degradation

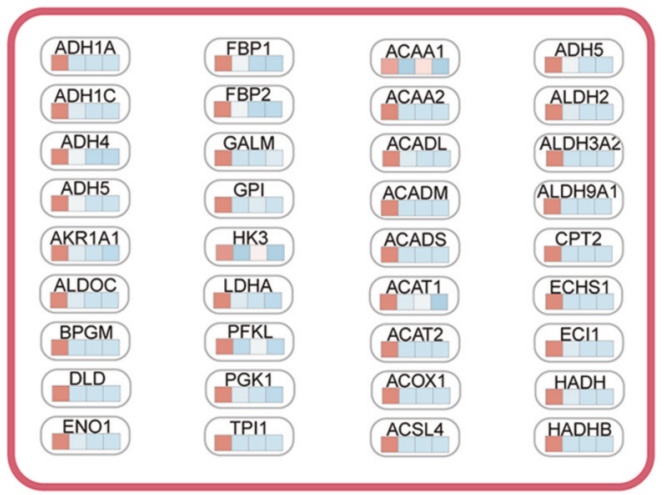

Salivary secretion \& Sphingolipid signaling \& Apoptosis pathway

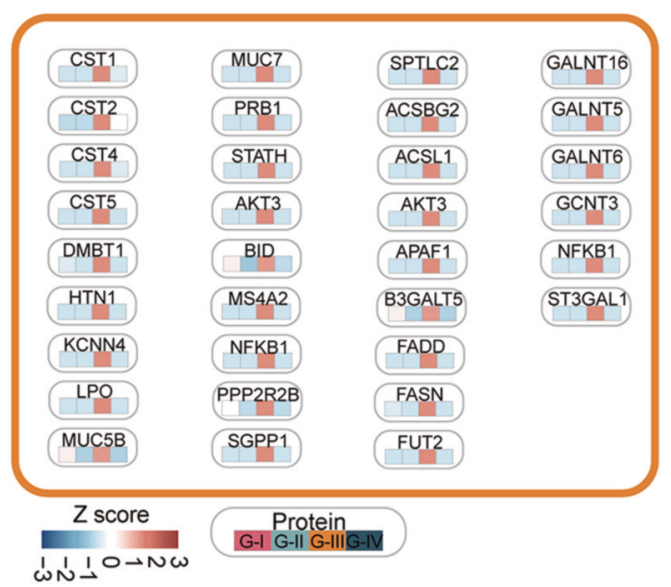

Pathway enrichment

Sphingolipid metabolism Protein digestion and absorption -

Glycosaminoglycan degradation ECM-receptor interaction

Cell adhesion molecules (CAMs) -

Antigen processing and presentation-

Sphingolipid signaling pathway

Mucin type O-Glycan biosynthesis -

Glycosphingolipid biosynthesis - globo series

Fatty acid biosynthesis -

Systemic lupus erythematosus -
Apoptosis -

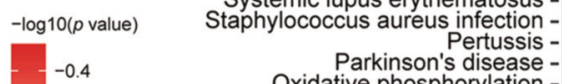

Parkinson's disease -

Oxidative phosphorylation -

ECM-receptor adhesion

Complement and coagulation cascades -

Proteasome -

Phagosome -

Pentose phosphate pathway

Metabolic pathways -

Glyoxylate and dicarboxylate metabolism -

Glycolysis / Gluconeogenesis -
Fatty acid degradation -
Carbon metabolism -

Biosynthesis of antibiotics -

Biosynthesis of amino acids -

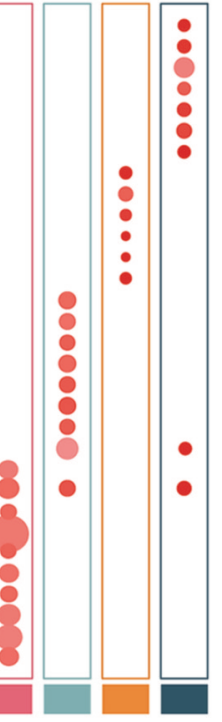

Complement and coagulation cascades \& ECM

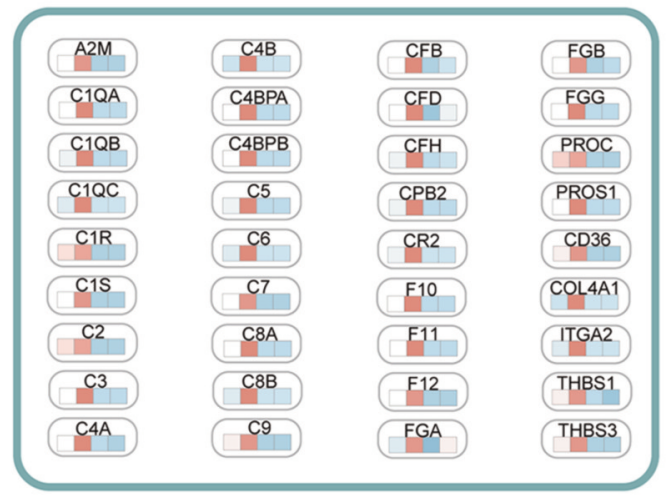

Lysosome \& Cell adhesion molecules (CAMs)

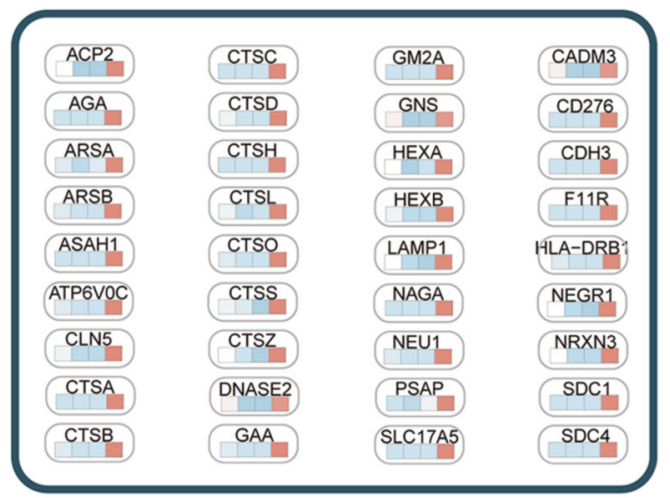

Figure 3. Proteomic clustering of ten body fluids. (A) The consensus clustering analysis of ten body fluids identified four clusters. The heatmap depicts the relative abundance of the signature proteins in four subtypes $(Z$ score of $i B A Q)$. (B) A bubble plot of the KEGG pathway enrichment of $C-I, C-I I, C-I I I$, and C-IV is shown. (C) Diagram summarizes the differentially expressed signatures and signaling cascades involved in C-I to $C$ $I V$. The heatmap under each protein depicts the $Z$ score of average protein abundance in each proteomic subtype. CSF: Cerebrospinal fluid; KJF: knee-joint fluid; ECM: extracellular matrix; KEGG: Kyoto Encyclopedia of Genes and Genomes; TCA: tricarboxylic acid cycle. 
Table II. The KEGG pathways of four clusters.

\begin{tabular}{|c|c|c|}
\hline Term & Count & $p$-Value \\
\hline \multicolumn{3}{|l|}{ The KEGG pathways of cluster C-I } \\
\hline hsa01130: Biosynthesis of antibiotics & 96 & $1.64 \mathrm{E}-24$ \\
\hline hsa03050: Proteasome & 37 & $3.80 \mathrm{E}-22$ \\
\hline hsa01200: Carbon metabolism & 60 & $1.23 \mathrm{E}-19$ \\
\hline hsa01100: Metabolic pathways & 296 & $6.34 \mathrm{E}-18$ \\
\hline hsa00010: Glycolysis / Gluconeogenesis & 37 & $6.47 \mathrm{E}-13$ \\
\hline hsa01230: Biosynthesis of amino acids & 37 & $1.07 \mathrm{E}-11$ \\
\hline hsa04145: Phagosome & 54 & $2.33 \mathrm{E}-09$ \\
\hline hsa00071: Fatty acid degradation & 24 & $6.20 \mathrm{E}-09$ \\
\hline hsa00030: Pentose phosphate pathway & 19 & $1.95 \mathrm{E}-08$ \\
\hline hsa00630: Glyoxylate and dicarboxylate metabolism & 17 & $3.06 \mathrm{E}-07$ \\
\hline \multicolumn{3}{|l|}{ The KEGG pathways of cluster C-II } \\
\hline hsa04610: Complement and coagulation cascades & 49 & $1.24 \mathrm{E}-53$ \\
\hline hsa05150: Staphylococcus aureus infection & 18 & $5.94 \mathrm{E}-12$ \\
\hline hsa05322: Systemic lupus erythematosus & 23 & 4.88E-09 \\
\hline hsa05012: Parkinson's disease & 23 & $1.48 \mathrm{E}-08$ \\
\hline hsa05133: Pertussis & 15 & $6.67 \mathrm{E}-07$ \\
\hline hsa04512: ECM-receptor interaction & 15 & 4.27E-06 \\
\hline hsa00190: Oxidative phosphorylation & 18 & $1.03 \mathrm{E}-05$ \\
\hline hsa04510: Focal adhesion & 22 & $3.31 \mathrm{E}-05$ \\
\hline hsa04145: Phagosome & 18 & $5.04 \mathrm{E}-05$ \\
\hline \multicolumn{3}{|l|}{ The KEGG pathways of cluster C-III } \\
\hline hsa04970: Salivary secretion & 12 & $3.84 \mathrm{E}-07$ \\
\hline hsa00512: Mucin type O-Glycan biosynthesis & 5 & 0.002302219 \\
\hline hsa04071: Sphingolipid signaling pathway & 7 & 0.022467508 \\
\hline hsa00061: Fatty acid biosynthesis & 3 & 0.022752444 \\
\hline hsa00603: Glycosphingolipid biosynthesis - globo series & 3 & 0.026230468 \\
\hline hsa04210: Apoptosis & 5 & 0.026462612 \\
\hline \multicolumn{3}{|l|}{ The KEGG pathways of cluster C-IV } \\
\hline hsa04142: Lysosome & 39 & $3.98 \mathrm{E}-28$ \\
\hline hsa00531: Glycosaminoglycan degradation & 8 & $1.24 \mathrm{E}-06$ \\
\hline hsa04514: CAMs & 14 & 6.61E-04 \\
\hline hsa04512: ECM-receptor interaction & 10 & 0.001916782 \\
\hline hsa04974: Protein digestion and absorption & 9 & 0.007445093 \\
\hline hsa00600: Sphingolipid metabolism & 6 & 0.017356452 \\
\hline hsa04145: Phagosome & 11 & 0.023555688 \\
\hline hsa04610: Complement and coagulation cascades & 7 & 0.023805229 \\
\hline hsa04612: Antigen processing and presentation & 7 & 0.036092838 \\
\hline
\end{tabular}

KEGG: Kyoto Encyclopedia of Genes and Genomes, ECM: extracellular matrix; CAMs: cell adhesion molecules.

function (Figure 4E and Table III). For example, the specific proteins identified in ascites and bile were separately clustered into pancreatic secretion ( $p=9.83 \mathrm{E}-4)$ (such as PNLIPRP1, PNLIPRP2, CPA2, CELA2A, and CELA3B) and bile secretion $(p=1.33 \mathrm{E}-6)$ (such as SCARB1, SLC5A1, ATP1B1, BAAT, and AQP1). Ascites was additionally enriched for long-term potentiation ( $p=1.49 \mathrm{E}-2)$ (e.g. PPP3R1, MAP2K2, PLCB2, etc.), chemokine signaling pathway ( $p=2.17 \mathrm{E}-2)$ (e.g. ROCK1, GRK6, GNB4, etc.), and platelet activation ( $p=2.47 \mathrm{E}-2)(e . g$. APBB1IP, SYK, COL5A3, etc.). Bile was enriched for metabolic pathways ( $p=5.34 \mathrm{E}-5)$, such as histidine metabolism $(p=1.13 \mathrm{E}-3)$ (e.g. ALDH3A2, MAOA, HNMT, etc.) and fat digestion and absorption ( $p=1.47 \mathrm{E}-2)$ (e.g. ABCG8, FABP2, NPC1L1, etc.). CSF was enriched for cell adhesion molecules 
(CAMs) ( $p=5.20 \mathrm{E}-4)$, including NFASC, CDH4, NRXN1, NRXN2, CNTN2, and LRRC4B. Plasma was characterized with oxidative phosphorylation ( $p=6.74 \mathrm{E}-6)$ (e.g. NDUFA8, UQCRB, ATP5I, etc.), mismatch repair and DNA replication ( $p=1.33 \mathrm{E}-2,3.11 \mathrm{E}-2$, respectively) (e.g. RPA1, RPA2, SSBP1, etc.), and tight junction $(p=2.91 \mathrm{E}-2)(e . g$. ACTN2, MYH8, ACTG1, etc.). As expected, salivary secretory proteins, including MUC7, KCNN4, CST5, and HTN1, were specifically overrepresented in saliva. In addition, we found that the MAPK signaling pathway ( $p=1.05 \mathrm{E}-2)$ (e.g. MAP2K3, EGF, NFKB1, etc.) was enriched in saliva, while apoptotic process and cell proliferation related bioprocesses were also significantly enriched $(p<0.05)$. Some vitamin digestion- and absorptionrelated proteins (such as CUBN, LMBRD1, and RBP2) were identified in urine. In addition, tumor necrosis factor-mediated signaling related proteins (such as TNFRSF12A, CD27, RELT, and PSMA8) were also highly expressed in urine.

The differential proteins and features of 10 body fluids. A total of 304 proteins ( $9.0 \%$ of all identified proteins) were identified in all analyzed body fluids, which may indicate that these proteins were essential for various life activities. Accordingly, most of these common proteins in body fluids were complement and coagulation components (such as $\mathrm{C} 3, \mathrm{C} 4 \mathrm{~A}, \mathrm{CPB} 2, \mathrm{CFB}$, and FGB), apolipoproteins (such as $\mathrm{CDC} 42$, APOA1, and APOA2), and filament tropomyosin (such as TPM1, TPM2, DMTN, EPB41, and ANK1), which may partially be due to the material exchange among body fluids. The commonly identified proteins were functionally categorized based on universal KEGG pathway terms (Figure 5A). The top annotated pathways included focal adhesion ( $p=7.12 \mathrm{E}-6)$ (e.g. LAMA2, EGFR, THBS3, etc.), complement and coagulation cascades $(p=3.32 \mathrm{E}-$ 5) (e.g. FGB, C3, C4A, etc.), biosynthesis of amino acids $(p=4.69 \mathrm{E}-5)$ (e.g. PKM, TPI1, CPS1, etc.), ECM-receptor interaction $(p=2.08 \mathrm{E}-4)$ (e.g. LAMA2, COL5A1, COL6A2, etc.), biosynthesis of antibiotics ( $p=5.04 \mathrm{E}-4)$ (e.g. PGM1, PFKP, BCAT2, etc.), and lysosome ( $p=5.86 \mathrm{E}-4)$ (e.g. AGA, CTSD, CTSS, etc.). Metabolic pathways such as carbon metabolism ( $p=5.40 \mathrm{E}-3)$, vitamin digestion and absorption $(p=1.47 \mathrm{E}-2)$ and glycolysis/gluconeogenesis $(p=2.13 \mathrm{E}-2)$ were also highly activated.

The above enrichment results enabled us to classify the data into categories by a cluster analysis (Figure 5B). Based on an unsupervised hierarchical clustering analysis, the plasma and serum (S-I), hydrothorax and KJF (S-II), saliva and tears (S-III), CSF and urine (S-IV), ascites and bile (S-V), were separately clustered into five subgroups; the five subgroups were identified as significantly different compared with each other. Furthermore, the KEGG pathway enrichment analysis showed the most dominant features among the five subgroups. For example, complement and coagulation cascades $(p=9.53 \mathrm{E}-8)$, lysosome $(p=1.10 \mathrm{E}-4)$, and metabolic pathways $(p=2.61 \mathrm{E}-2)$ were separately dominant in S-I, S-IV, and S-V (Figure 5C and Table IV). We further explored the main functional feature of body fluids corresponding to one subgroup. The KEGG enrichment analysis showed that in the S-IV subgroup, urine was associated with lysosome ( $p=8.10 \mathrm{E}-6)$ and sphingolipid metabolism ( $p=5.82 \mathrm{E}-3)$, while in CSF, CAMs $(p=1.71 \mathrm{E}-2)$ were enriched. In another example, in subgroup $\mathrm{S}-\mathrm{V}$, ascites was associated with metabolism-related pathways, such as biosynthesis of amino acids $(p=1.90 \mathrm{E}-3)$, biosynthesis of antibiotics ( $p=6.98 \mathrm{E}-4)$, carbon metabolism $(p=6.79 \mathrm{E}-3)$, and glycolysis/gluconeogenesis ( $p=2.25 \mathrm{E}-2)$; bile was characterized by protein processing in endoplasmic reticulum $(p=1.48 \mathrm{E}-2)$ and proteasome ( $p=2.55 \mathrm{E}-2)$ (Figure 5D and Table IV). The features of body fluids were consistent with the features of the respective subgroup. In Figure 5E, the overrepresented proteins of 10 body fluids are listed; C3, C4A, CFB, EGFR, and ILK were highly expressed in plasma; AGA, ARSA, ASAH1, CTSD, and GLB1 were enriched in urine.

For the 1,683 proteins (49.5\% of all identified proteins), which were identified in at least two body fluids, we performed the same unsupervised hierarchical clustering analysis, and arrived at a similar clustering result, of which saliva and tears, hydrothorax and KJF, CSF and urine, and plasma and serum, were clustered into one subgroup, respectively (Figure 6A). Regarding these proteins, the KEGG enrichment result revealed the differential functions of each body fluid. Specifically, urine-enriched proteins were mainly related to lysosome, saliva-enriched proteins were mainly involved in salivary secretion, ascites-enriched and bile-enriched proteins were important in metabolic bioprocesses, hydrothorax-enriched proteins were mainly involved in platelet activation and focal adhesion pathways, while plasma-enriched proteins in the immune and complement system.

Figure 4. Correlation analysis among ten body fluids and their specific protein components and functional features. (A) The matrix of correlation plots revealed a highly varied correlation between the proteins' intensities in ten body fluids. (B) Venn diagram showing proteins that were differentially expressed in these four body fluids (ascites, KJF, hydrothorax, and plasma); protein numbers and proportions in these body fluids are also shown. (C) Principal component analysis (PCA) of the proteome pattern in ten body fluids. (D) Pie chart showing protein identification among ten body fluids. (E) The heatmap depicts the relative abundance of the signature proteins in each body fluid ( $Z$ score of $i B A Q$ ) (above). A bubble plot of the KEGG pathway enrichment of each body fluid is shown (below). CSF: Cerebrospinal fluid; KJF: knee-joint fluid; ESCRT: Endosomal Sorting Complex Required for Transport; MAPK: mitogen-activated protein kinase; JAK-STAT: janus kinase-signal transducer and activator of transcription; KEGG: Kyoto Encyclopedia of Genes and Genome. 
A

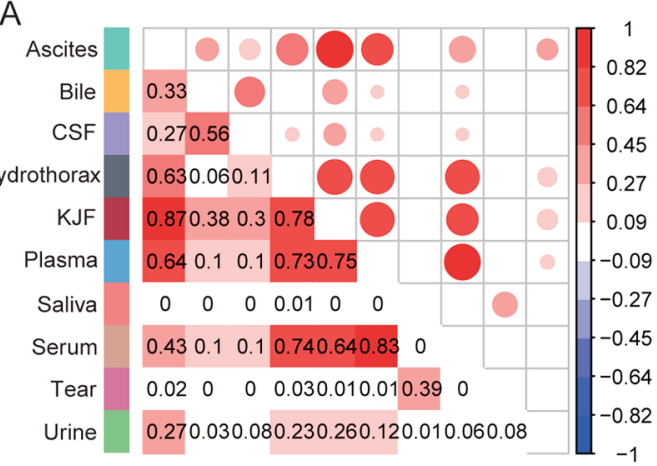

D
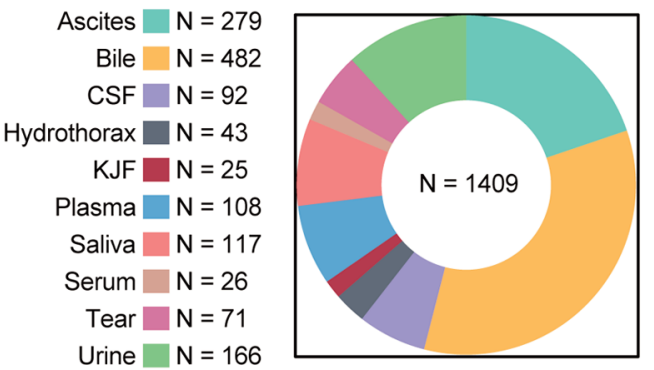
Vitamin digestion and absorption-
Viral budding via host ESCRT complexTumor necrosis factor-mediated signaling pathwayMuscle contraction Response to copper ion-
Regulation of cardiac muscle contraction by calcium ion signaling Metabolic process Pyruvate metabolismBiotin metabolic processSalivary secretion MAPK signaling pathwayApoptotic process Apoptotic process Oxidative phosphorylation Non-alcoholic fatty liver disease (NAFLD)Mismatch repair -
Homologous recombination DNA replicationneuromuscular junction development-
Defense responseSexual reproductionPositive regulation of JAK-STAT cascadeCellular protein metabolic process Skeletal system developmentcentral nervous system developmentCell adhesion molecules (CAMs)-

Cell adhesion molecules (CAMs) Cell adhesion-

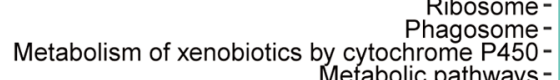
Metabolic pathways Histidine metabolismFat digestion and absorption -
Bile secretion ABC transportersSynaptic vesicle cyclePlatelet activation -

Pancreatic secretion -

Long-term potentiation Endocytosis-

Endocrine and other factor-regulated calcium reabsorptionChemokine signaling pathway-
C

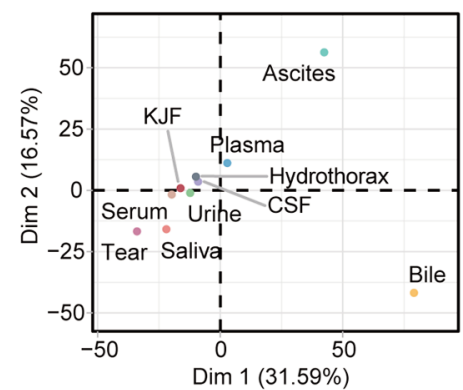

E
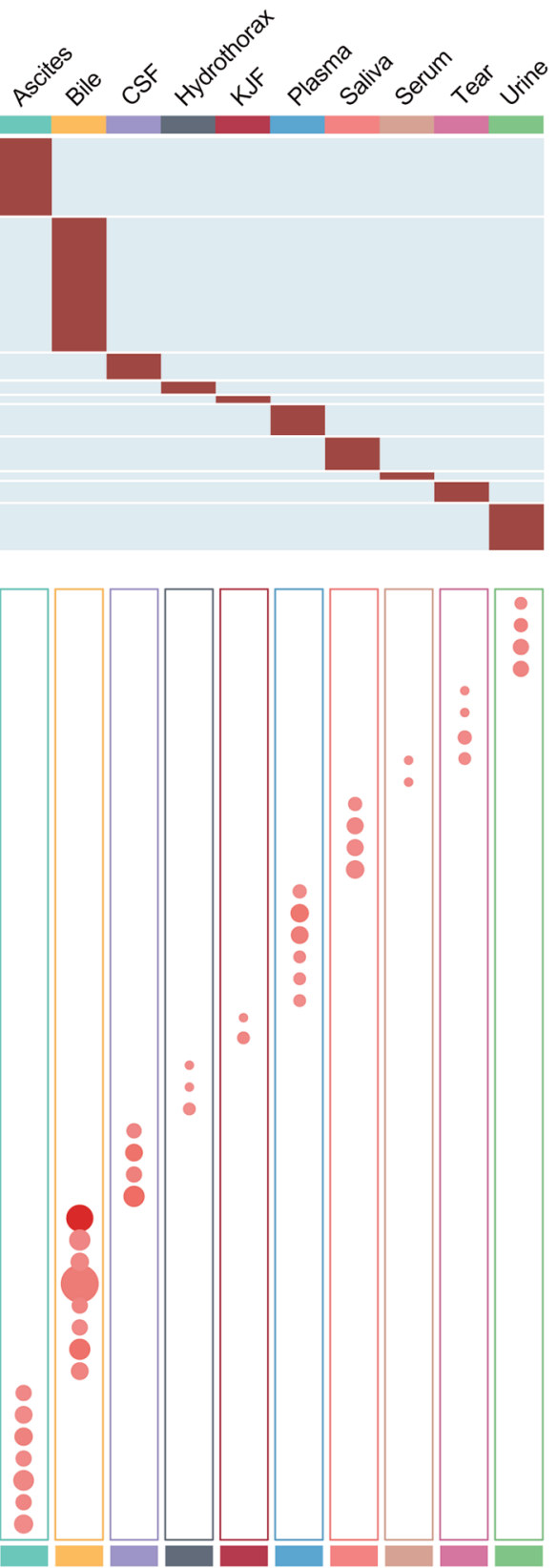

Ascites

Bile

CSF

Hydrothorax

KJF

Plasma

Saliva

Serum

Tear

Urine

\begin{tabular}{ll}
3 \\
2 \\
힝 & 1 \\
\hline & 0
\end{tabular}

巡 0

$\begin{array}{ll}N & -1 \\ -2 \\ -3\end{array}$

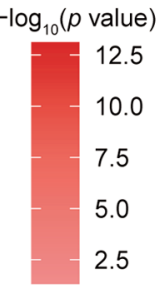

Gene

- 20

- 40

? 60 
The possible connection between different body fluids. Blood (plasma or serum) plays a central role in circulation, while the other 8 body fluids might connect with blood directly or indirectly. The comparisons between plasma and other 9 body fluids revealed that the overlap between plasma and KJF was the second high (87.0\%) after serum $(91.5 \%)$ (Figure 6B). In addition, we also found the hydrothorax, tears, and CSF showed a larger overlap proportion with plasma $(77.7 \%, 74.7 \%$, and $72.0 \%$, respectively). The KEGG pathways of plasma and CSF were similar to each other, mainly the ones related to immune function (complement and coagulation cascades, and platelet activation) (Figure 6A). As shown in Figure 6C, a model was built for illustrating the possible connection between different body fluids based on previous reports (37) and the results in this study. The protein compositions and functional annotations showed that the plasma, hydrothorax, and KJF were more similar than the other fluids, suggesting that a great deal of material exchanges occurred among them.

\section{Discussion}

Human body fluids have been an important material for disease diagnosis, having many advantages including minimum cost, noninvasiveness, and ease of sample collection. Therefore, measuring biomolecules in body fluids or tissues for diagnosis, monitoring, and prognostic purposes is key to help clinicians and guide decision making for treatment, patient management, and the development of drugs and prevention strategies. Recently, several large-scale proteomic studies of body fluids were conducted. The Plasma Proteome Database (PPD; http://www.plasmaproteomedatabase.org/) (38, 39) contains information on 10,546 proteins detected in serum/plasma linked to 509 scientific articles, 3,784 of which were reported in at least two research articles in the 2014 updated version. In addition, Mann's group reported that the human urinary proteome contains more than 1,500 proteins which include a large proportion of membrane proteins. The latest largestscale human CSF proteome identified 3,256 nonredundant proteins (40). Compared with plasma/serum, urine, CSF, saliva, bile, tears and ascites, the proteomes of hydrothorax and KJF are less studied. Even though the open-access online repositories of some human body fluids such as plasma (http://www.plasmaproteomedatabase.org/) (38, 39), urine (http://urineproteomics.org/databases.html) (41), and saliva (https://www.jcvi.org/research/saliva-proteome-database) (42), have been described. The characterization of sample matrices taken from other human body fluids is still not fully comprehensive.

With the development of LC-MS/MS technology, protein biomarker discovery has become one of the main applications of proteomics. Considering the advantages of body fluids, analysis of human body fluid proteomes has become one of the most promising approaches to discover biomarkers for human diseases.

Here, we performed a label-free proteomic analysis on ten body fluids from 23 patients diagnosed with 19 common diseases, and identified a total of 3,396 nonredundant proteins. The body fluids in the study included ascites, bile, CSF, hydrothorax, KJF, plasma, saliva, serum, tear fluid and urine. In our study, we found 1,374, 688, 116, 331, 1,168, 369, 337 and 495 proteins in ascites, bile, CSF, KJF, plasma, saliva, tear fluid and urine, respectively, that were not reported in previously published proteomic datasets of body fluids (Figure 7A). Nevertheless, some of the proteins that we identified had also been included in previous studies. Specifically, Jin et al. identified 4,856 specific proteins in the ascitic fluids from gastric cancer; 491 of these proteins were also detected in ascites, in the present study (43). Farina et al. identified 1,678 bile-specific proteins, 1,421 of which were common with our results (44). Macron et al. identified 3,379 proteins in CSF samples from normal human. Compared with normal CSF proteome, our study identified 872 commonly shared proteins (such as OMG, SNCA, APOA1, APOE, APOM) in the CSF (45). The additional 116 proteins specifically identified in our study might provide potential biomarkers for the diagnosis of subarachnoid hemorrhage (SAH), chair deformity with syringomyelia, and the right cerebellar hemangioblastoma. Bhattacharjee et al. identified 870 proteins in total in synovial fluid and among them, 456 proteins overlapped with the proteome of KJF in our study (46). Nanjappa et al. updated the PPD in 2014 and identified 10,546 proteins of plasma in total; among them, 109 proteins were also identified in the current study. Compared with the published saliva proteome data, our study identified 458 overlapping proteins, which accounted for $15.7 \%$ of the 2,462 proteins identified by Ai et al. in 2010 (47). Mann's group researchers, de Souza et al. and Adachi et al. identified 491 and 1,500 proteins in tear fluid proteome (48) and urinary proteome (22) of healthy donors separately, of which, 122 and 628, respectively, were commonly identifiable in our study. Compared with the published proteomic data, 495 urinary proteins were specific

Figure 5. The differential features of common proteins identified in ten body fluids. (A) Bar plots depict KEGG pathways of commonly identified proteins among ten body fluids. (B) Hierarchical clustering based on the $i B A Q$ quantification. (C) A bubble plot of the KEGG pathway enrichment of S-I, S-II, S-III, S-IV and $S$-V is shown. (D) A bubble plot of the KEGG pathway enrichment of some body fluids is shown. (E) The line charts of overrepresented proteins of ten body fluids. PPAR: Peroxisome proliferator-activated receptor; ECM: extracellular matrix; CSF: cerebrospinal fluid; KJF: knee joint fluid; MAPK: mitogen-activated protein kinase; KEGG: Kyoto Encyclopedia of Genes and Genome. 
A

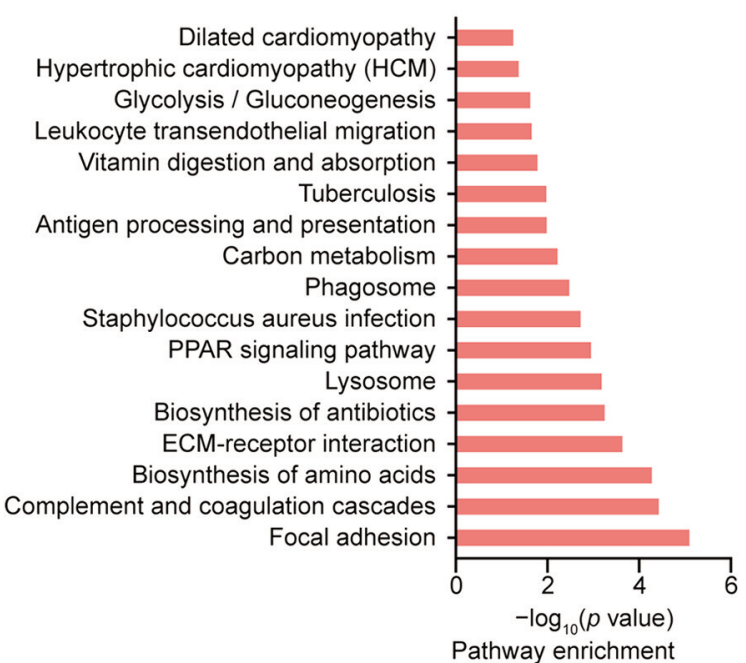

C

Metabolic pathways MAPK signaling pathway Glycolysis / Gluconeogenesis Carbon metabolism Biosynthesis of antibiotics Biosynthesis of amino acids Sphingolipid metabolism Lysosome -

Nitrogen metabolism Leukocyte transendothelial migration Rheumatoid arthritis ECM-receptor interaction Staphylococcus aureus infection Regulation of actin cytoskeleton PPAR signaling pathway Focal adhesion Complement and coagulation cascades -

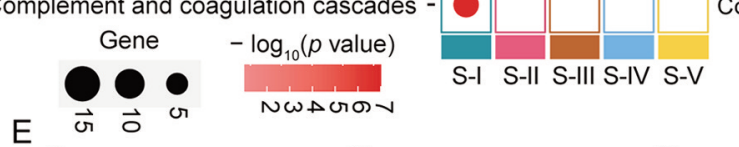

E
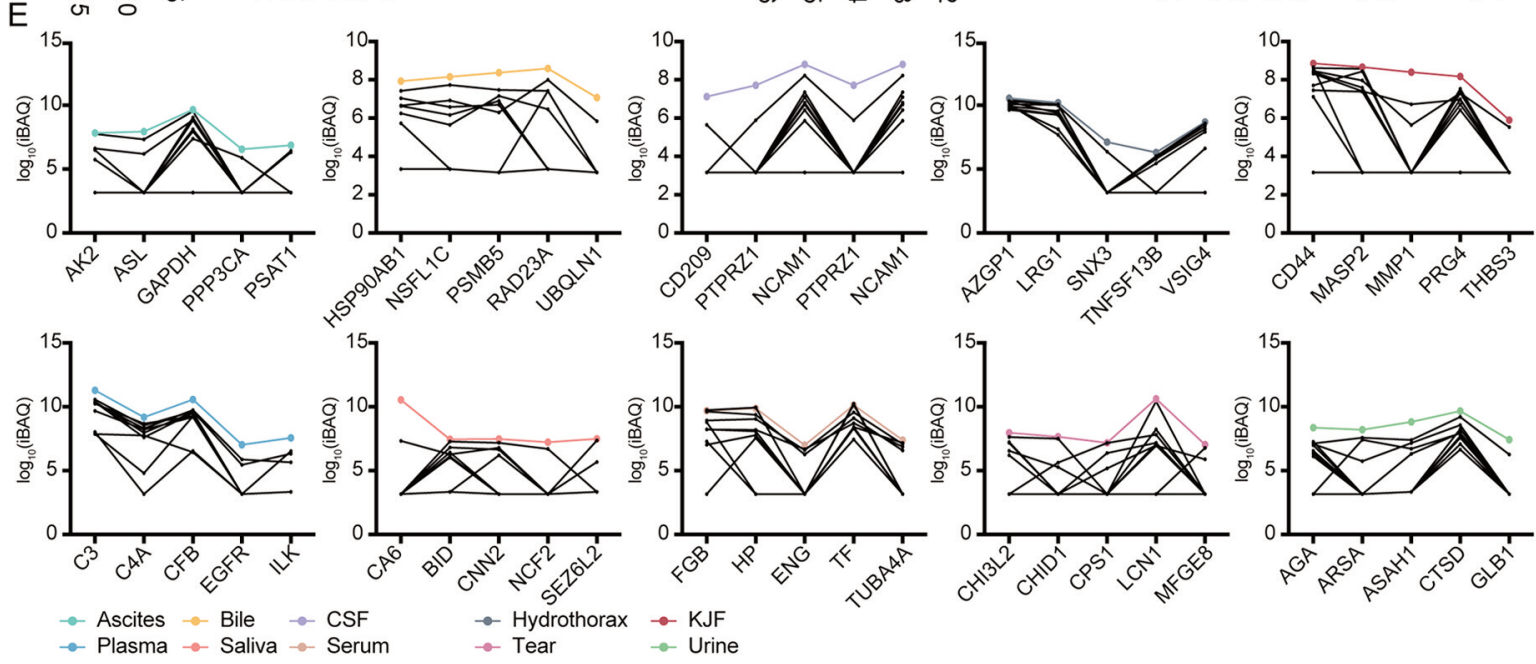

B

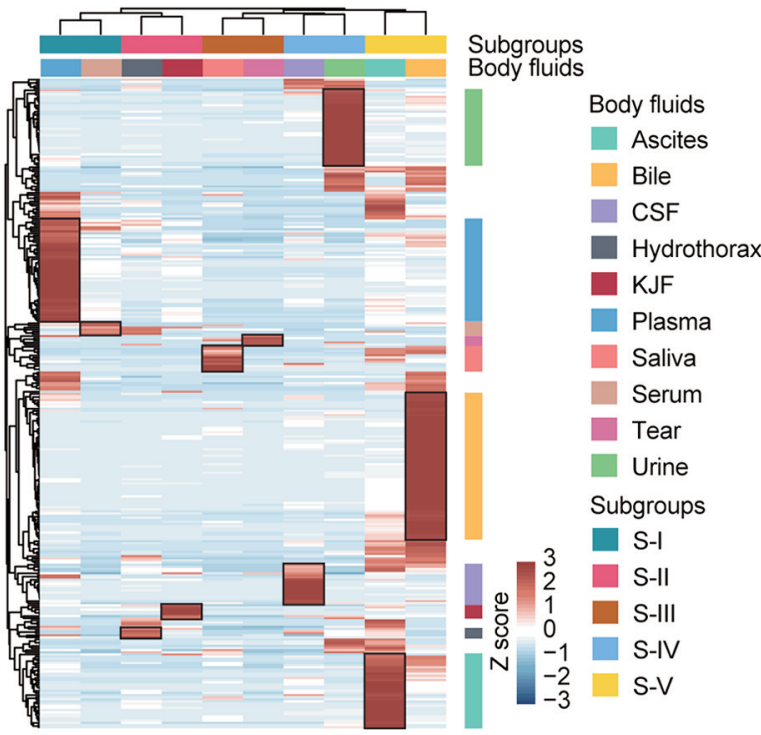

D

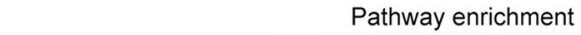

Protein processing in Proteasome -

Glycolysis / Gluconeogenesis -

Carbon metabolism -

Biosynthesis of antibiotics -

Biosynthesis of amino acids -

Sphingolipid metabolism -

Other glycan degradation -

Lysosome -

Cell adhesion molecules (CAMs) Leukocyte transendothelial migration -

ECM-receptor interaction -

Staphylococcus aureus infection -

Regulation of actin cytoskeleton -

PPAR signaling pathway Focal adhesion -

Complement and coagulation cascades -

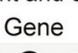

$-\log _{10}(p$ value $)$

-

$\omega \omega \mathrm{G}$

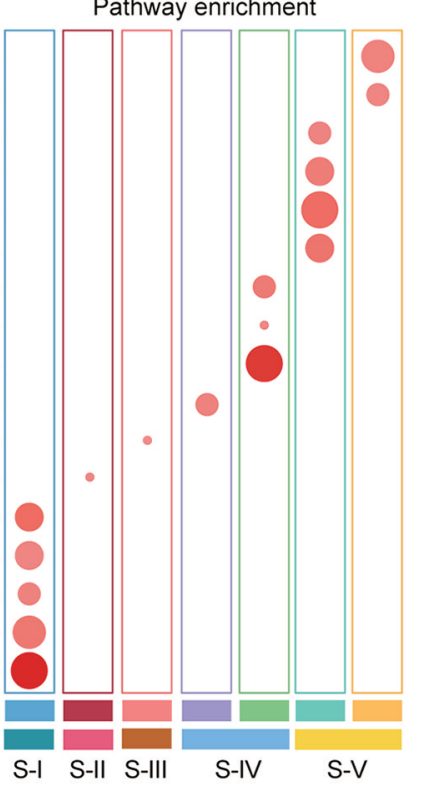


Table III. The KEGG/GO-BP pathways of body fluid-specific proteins detected in each body fluid.

\begin{tabular}{lcc}
\hline Term & Count & $p$-Value \\
\hline The KEGG pathways of the ascites-specific proteins & & \\
\hline hsa04972: Pancreatic secretion & 9 & $9.83 \mathrm{E}-04$ \\
hsa04961: Endocrine and other factor-regulated calcium reabsorption & 6 & 0.00295514 \\
hsa04144: Endocytosis & 13 & 0.006887314 \\
hsa04721: Synaptic vesicle cycle & 6 & 0.012388033 \\
hsa04720: Long-term potentiation & 10 & 0.014945923 \\
hsa04062: Chemokine signaling pathway & 8 & 0.021716756 \\
hsa04611: Platelet activation & 8 & 0.024694693 \\
\hline
\end{tabular}

The KEGG pathways of the bile-specific proteins

hsa03010: Ribosome

hsa04976: Bile secretion

hsa01100: Metabolic pathways

hsa00340: Histidine metabolism

hsa02010: ABC transporters

hsa04145: Phagosome

hsa00980: Metabolism of xenobiotics by cytochrome P450

hsa04975: Fat digestion and absorption

$\begin{array}{rc}29 & 7.67 \mathrm{E}-14 \\ 14 & 1.33 \mathrm{E}-06 \\ 72 & 5.34 \mathrm{E}-05 \\ 6 & 0.001131805 \\ 8 & 0.001147674 \\ 14 & 0.004152053 \\ 9 & 0.006359228 \\ 6 & 0.014711489\end{array}$

The KEGG/GO-BP pathways of the CSF-specific proteins

hsa04514: CAMs

GO:0007155 - cell adhesion

GO:0007417 - central nervous system development

GO:0001501 - skeletal system development

The GO-BP pathways of the hydrotharox-specific proteins

GO:0019953 - sexual reproduction

GO:0044267 - cellular protein metabolic process

GO:0046427 - positive regulation of JAK-STAT cascade

$\begin{array}{rc}6 & 5.20 \mathrm{E}-04 \\ 14 & 3.22 \mathrm{E}-07 \\ 8 & 1.95 \mathrm{E}-06 \\ 5 & 0.004536909\end{array}$

The GO-BP pathways of the KJF-specific proteins

GO:0006952 - defense response

GO:0007528 - neuromuscular junction development

3

0.002844721

20.032844474

The KEGG pathways of the plasma-specific proteins

hsa00190: Oxidative phosphorylation

hsa04932: NAFLD

hsa03430: Mismatch repair

hsa03440: Homologous recombination

hsa04530: Tight junction

hsa03030: DNA replication

$\begin{array}{cc}2 & 0.021822892 \\ 3 & 0.02778669 \\ 2 & 0.047399203\end{array}$

The KEGG/GO-BP pathways of the saliva-specific proteins

hsa04010: MAPK signaling pathway

hsa04970: Salivary secretion

GO:0006915 - apoptotic process

GO:0008283 - cell proliferation

$\begin{array}{lc}9 & 6.74 \mathrm{E}-06 \\ 8 & 1.41 \mathrm{E}-04 \\ 3 & 0.013287547 \\ 3 & 0.020705914 \\ 4 & 0.029071185 \\ 3 & 0.03105129\end{array}$

The KEGG/GO-BP pathways of the serum-specific proteins

hsa00620: Pyruvate metabolism

GO:0006768 - biotin metabolic process

2

0.040017534

0.017373395

Table III. Continued 
Table III. Continued

Term Count

$p$-Value

The KEGG/GO-BP pathways of the tear-specific proteins

hsa04071: Sphingolipid signaling pathway

GO:0008152 - metabolic process

GO:0010882 - regulation of cardiac muscle contraction by calcium ion signaling

GO:0046688 - response to copper ion

The KEGG/GO-BP pathways of the urine-specific proteins

hsa04977: Vitamin digestion and absorption

GO:0039702 - viral budding via host ESCRT complex

GO:0006936 - muscle contraction

GO:0033209 - tumor necrosis factor-mediated signaling pathway
0.046730571

0.016806047

0.022298654

0.04718562

$\begin{array}{lc}3 & 0.046730571 \\ 4 & 0.016806047 \\ 2 & 0.022298654 \\ 2 & 0.04718562\end{array}$

0.02237321
$8.78 \mathrm{E}-04$
0.002423384
0.003697041

0.02237321

0.002423384

60.003697041

KEGG: Kyoto Encyclopedia of Genes and Genomes, GO-BP: gene ontology-biological process; CSF: cerebrospinal fluid; KJF: knee joint fluid; CAM: cell adhesion molecules; JAK-STAT: janus kinase-signal transducer and activator of transcription; NAFLD: non-alcoholic fatty liver disease; MAPK: mitogen-activated protein kinase; ESCRT: endosomal sorting complex required for transport.

identified in our study, which might be associated with these diseases, such as hepatitis B cirrhosis, abdominal aortic aneurysm, and liver lesions.

We presented a comprehensive proteome landscape, and consensus clustering identified four clusters (C-I to C-IV) with distinct proteome features: C-I (glycolysis/gluconeogenesis and fatty acid degradation), C-II (complement/coagulation cascades and ECM), C-III (salivary secretion, sphingolipid pathway, and apoptosis) and C-IV (lysosome and cell adhesion molecules). The consensus clustering analysis revealed the high similarity of proteome profiles among body fluids within one cluster, such as ascites, bile, and CSF within C-I cluster (Figures 2 and $3 \mathrm{~A}$ ). The overlap among body fluids within one cluster is shown in Figure 7B, implying the exchange of many proteins among these body fluids. We determined the body fluidspecific proteins and biological function, which might be applicable for disease diagnosis. For example, bile-enriched proteins were mainly involved in bile secretion and salivaenriched proteins in salivary secretion. We also observed the obvious enrichment of metabolic pathways such as histidine metabolism and fat digestion/absorption in bile, revealing its metabolic function.

In addition, we made a multi-dimensional analysis based on the body fluid-common proteins. The hierarchical clustering analysis of the commonly identified proteins in ten body fluids revealed five subgroups, and the overlap of two body fluids within one subgroup (S-I to $\mathrm{S}-\mathrm{V}$ ) are shown in Figure 7C. The subgrouping of common proteins (304) in ten body fluids revealed the shared function; for example, the major biological function of plasma and serum was immunerelated complement/coagulation cascades, as expected. The functional analysis of the remaining 1,683 proteins demonstrated a similar function clustering.
Table IV. The KEGG pathways of five subgroups identified by hierarchical clustering analysis.

\begin{tabular}{lc}
\hline Term & $p$-Value \\
\hline The KEGG pathways of subgroup S-I & \\
\hline hsa04610: Complement and coagulation cascades & $9.53 \mathrm{E}-08$ \\
hsa05150: Staphylococcus aureus infection & $8.24 \mathrm{E}-04$ \\
hsa04510: Focal adhesion & 0.00518338 \\
hsa03320: PPAR signaling pathway & 0.022469123 \\
hsa04810: Regulation of actin cytoskeleton & 0.035406977
\end{tabular}

The KEGG pathways of subgroup S-II

hsa04512: ECM-receptor interaction

0.061674004 hsa05323: Rheumatoid arthritis 0.062364762

The KEGG pathways of subgroup S-III

hsa01100: Metabolic pathways

0.014741754

hsa00340: Histidine metabolism

0.096238935

The KEGG pathways of subgroup S-IV

hsa04142: Lysosome

$1.10 \mathrm{E}-04$

hsa00600: Sphingolipid metabolism

0.015426555

The KEGG pathways of subgroup S-V

hsa01230: Biosynthesis of amino acids

hsa01130: Biosynthesis of antibiotics

hsa01200: Carbon metabolism

hsa00010: Glycolysis/Gluconeogenesis

hsa01100: Metabolic pathways

hsa04010: MAPK signaling pathway

4.94E-05

$1.34 \mathrm{E}-04$

0.020613038

0.024121316

0.026082515

0.029483207

KEGG: Kyoto Encyclopedia of Genes and Genomes, PPAR: peroxisome proliferator-activated receptor; ECM: extracellular matrix; MAPK: mitogen-activated protein kinase. 
A

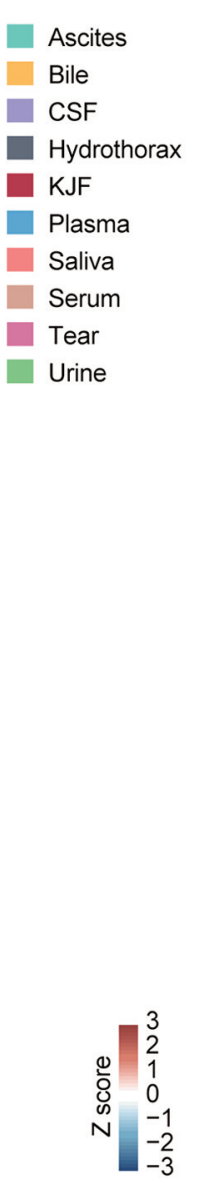

B

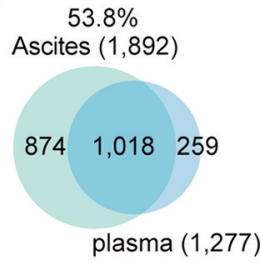

$77.7 \%$

Hydrothorax $(1,000)$

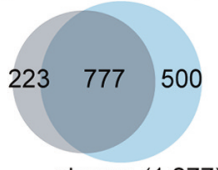

plasma $(1,277)$

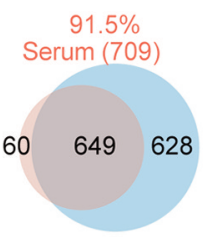

plasma $(1,277)$
$48.2 \%$

Bile $(2,109)$
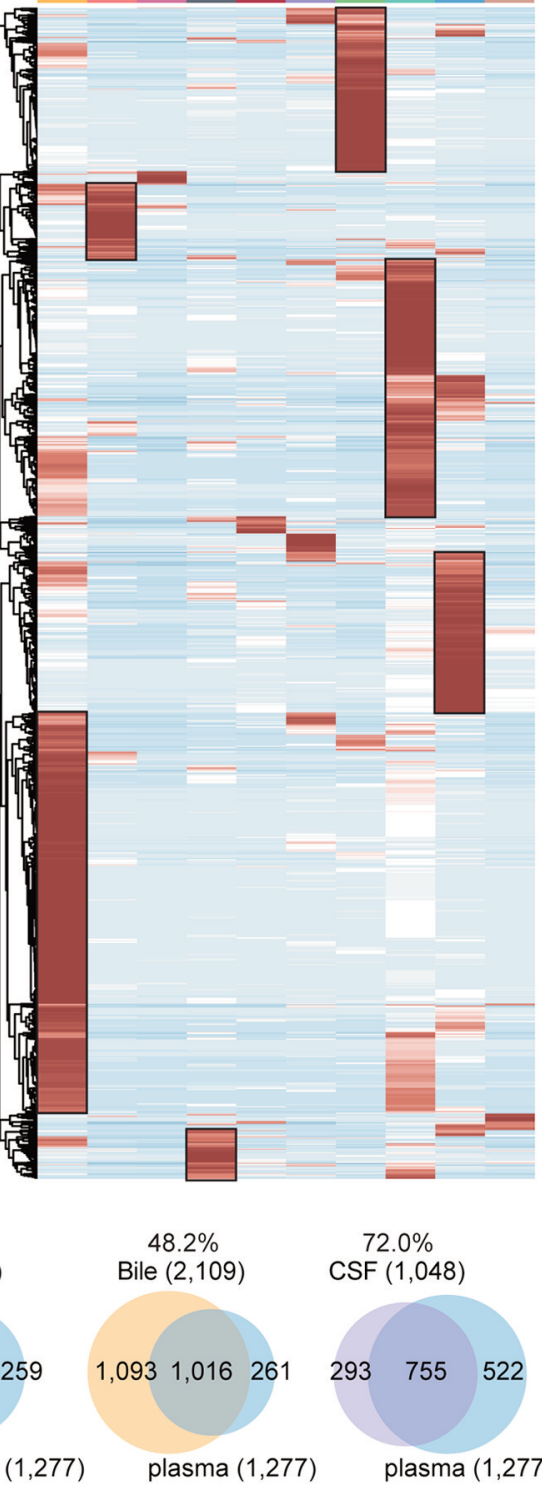

CSF $(1,048)$

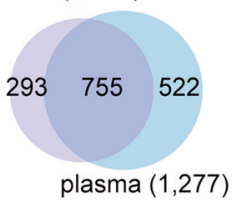

$63.1 \%$

Saliva (827)

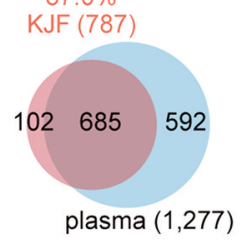

$$
74.7 \%
$$

Tear (459)

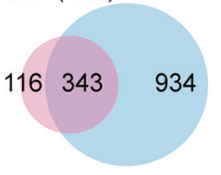

plasma $(1,277)$

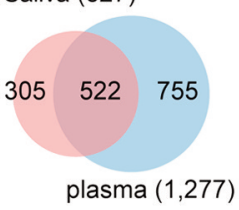

$59.4 \%$

Urine (1123)

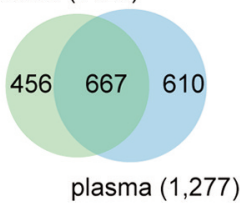

Pathway enrichment

Lysosome $(p$ value $=3.04 \mathrm{E}-19)$

Glycosaminoglycan degradation ( $p$ value $=4.75 \mathrm{E}-6$ )

PPAR signaling pathway $(\rho$ value $=2.33 E-3)$

Cell adhesion molecules (CAMs) $(p$ value $=1.41 \mathrm{E}-2)$

Salivary secretion $(p$ value $=2.91 \mathrm{E}-5)$

Protein processing in endoplasmic reticulum ( $p$ value $=6.72 \mathrm{E}-3$ )

Biosynthesis of antibiotics $(p$ value $=1.58 \mathrm{E}-10)$

Carbon metabolism ( $p$ value $=6.27 \mathrm{E}-7$ )

Fc gamma R-mediated phagocytosis ( $p$ value $=2.64 \mathrm{E}-6$ )

Pentose phosphate pathway ( $p$ value $=2.65 \mathrm{E}-6)$

Glycolysis / Gluconeogenesis ( $p$ value $=8.80 \mathrm{E}-6$ )

Bacterial invasion of epithelial cells $(p$ value $=3.88 \mathrm{E}-5$ )

ECM-receptor interaction $(p$ value $=1.08 \mathrm{E}-4)$

Focal adhesion $(p$ value $=1.21 \mathrm{E}-4)$

Complement and coagulation cascades ( $p$ value $=6.00 \mathrm{E}-42$ )

Staphylococcus aureus infection ( $p$ value $=3.17 \mathrm{E}-9)$

Systemic lupus erythematosus ( $p$ value $=1.66 \mathrm{E}-7$ )

Oxidative phosphorylation ( $p$ value $=1.82 \mathrm{E}-4)$

$\mathrm{ECM}$-receptor interaction $(\rho$ value $=1.17 \mathrm{E}-3)$

Proteasome ( $p$ value $=7.06 \mathrm{E}-18)$

Biosynthesis of antibiotics ( $p$ value $=3.86 \mathrm{E}-11)$

Carbon metabolism $(p$ value $=2.24 \mathrm{E}-8)$

Metabolic pathways $(p$ value $=7.87 \mathrm{E}-8)$

Glyoxylate and dicarboxylate metabolism ( $p$ value $=3.36 \mathrm{E}-6)$

Fatty acid degradation ( $p$ value $=3.93 \mathrm{E}-6$ )

Cysteine and methionine metabolism ( $p$ value $=1.04 \mathrm{E}-5)$

Pyruvate metabolism ( $p$ value $=1.71 \mathrm{E}-5)$

Glycolysis $/$ Gluconeogenesis $(p$ value $=1.87 \mathrm{E}-5)$

Glutathione metabolism ( $p$ value $=1.59 \mathrm{E}-4$ )

Biosynthesis of amino acids ( $p$ value $=1.84 \mathrm{E}-4$ )

Platelet activation ( $p$ value $=1.09 \mathrm{E}-5)$

Focal adhesion $(p$ value $=1.28 \mathrm{E}-3)$

Hydrothorax

C

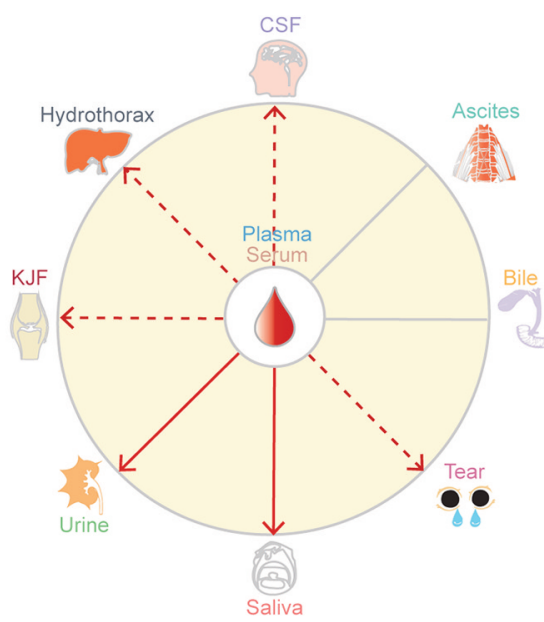

Figure 6. Possible association between the different body fluids. (A) Hierarchical clustering based on the $i B A Q$ quantification. (B) Venn diagram showing proteins that were differentially expressed in the plasma and other nine body fluids, the protein numbers and proportions in other nine body fluids are also shown. (C) The model illustrating the association among body fluids. The solid lines indicate known relationships among different body fluids, whereas dashed lines are used for our proposed relationships. CSF: Cerebrospinal fluid; KJF: knee joint fluid. 
A
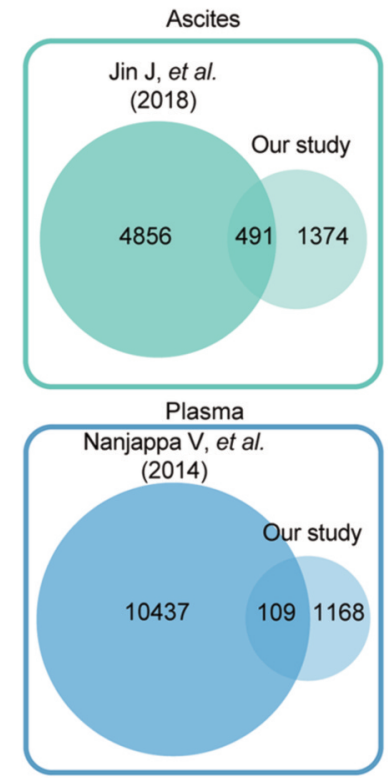

Bile

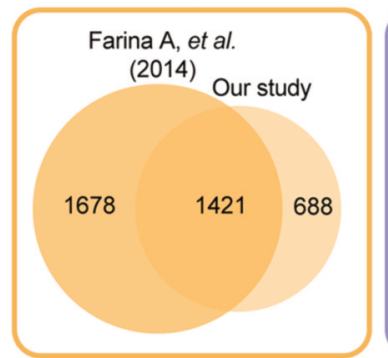

Saliva

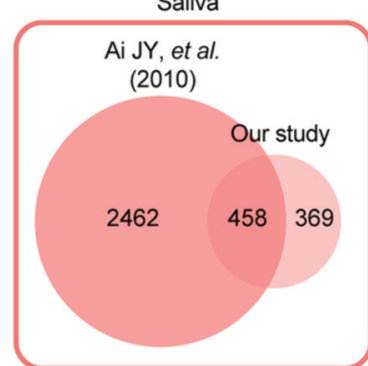

CSF
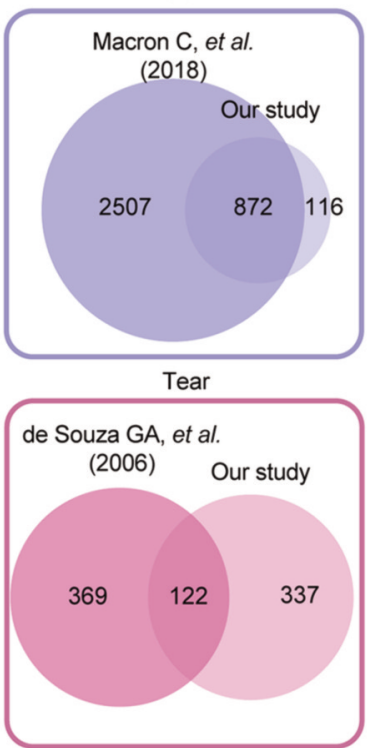

KJF
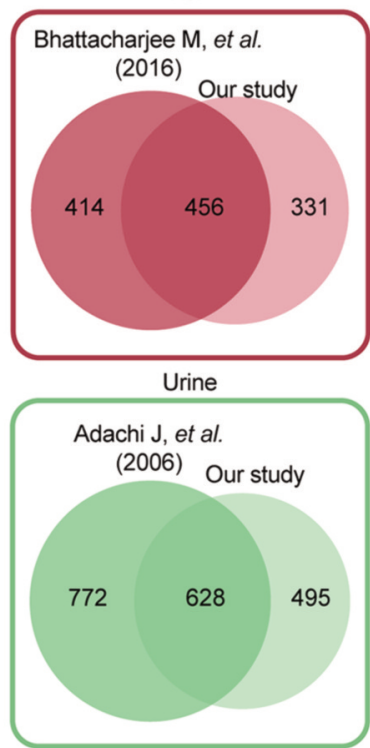

B

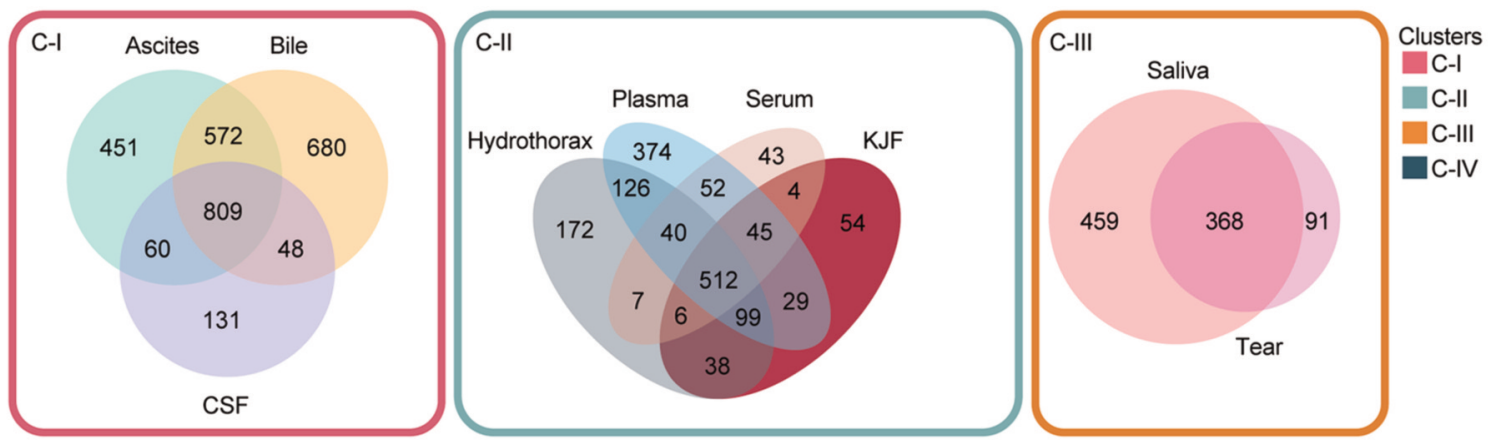

C

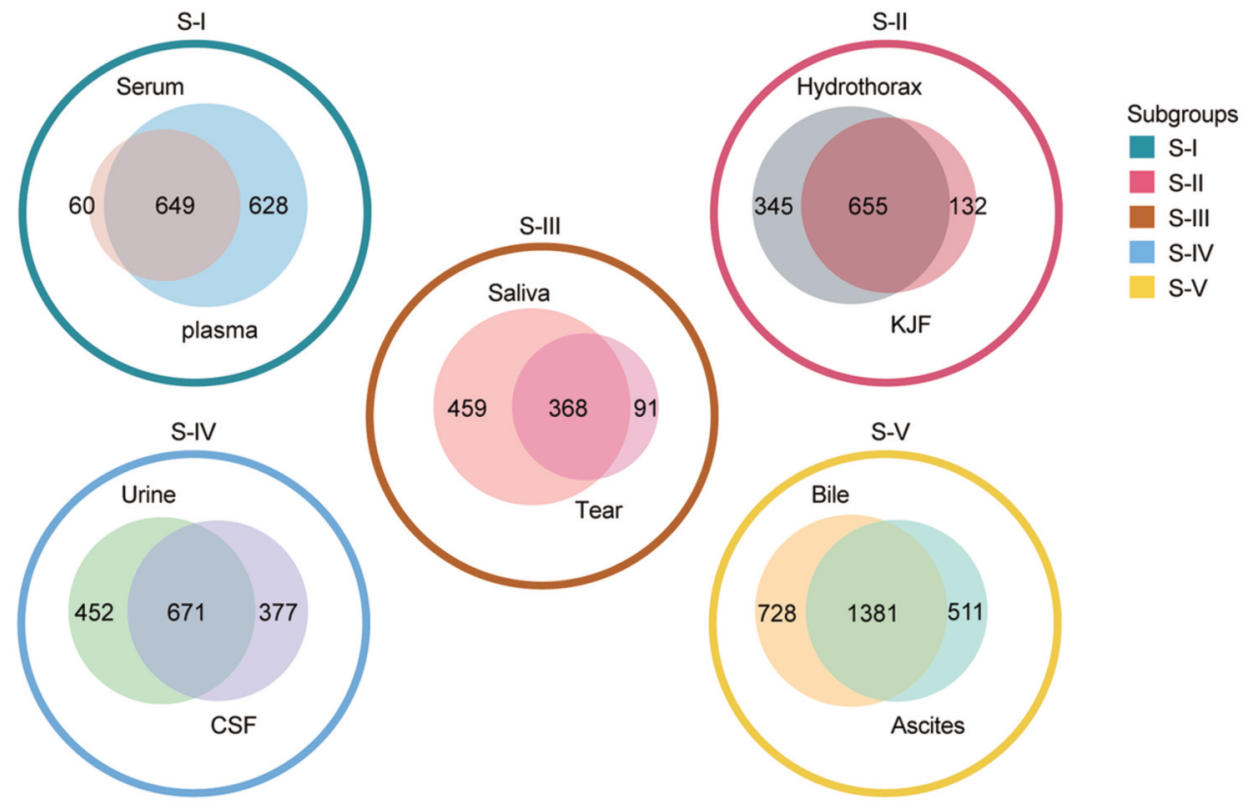

Figure 7. Venn diagram comparing the proteome of body fluids. (A) The protein datasets of eight body fluids including ascites, bile, CSF, KJF, plasma, saliva, tear and urine in this study were compared to the published proteome data of eight body fluids. (B) Venn diagram showing proteins differentially expressed in three clusters (C-I, C-II, and C-III); protein numbers and proportions are also shown. $(C)$ Venn diagram showing proteins differentially expressed in five subgroups (S-I, S-II, S-III, S-IV, and S-V); protein numbers and proportions are also shown. SCF: Cerebrospinal fluid; KJF: knee joint fluid. 
Table V. Common diseases and their associated body fluids markers.

Common diseases and their associated urine markers

\begin{tabular}{ll}
\hline Disease & Urine markers \\
\hline Arthritis & ACAN, ADAMTS1, ADAMTS2, ANXA5, BSG, CD5L, CILP, COMP, CRP, CSTA, FLG, GPI, HLA-DRB1, \\
& IL1RN, ITIH1, ITIH3, LCN2, MMP9, ORM2, PRTN3, S100A8, S100A9, TIMP1 \\
Myositis & CANX, DES, HLA-A, HLA-DRB1, ICAM1, KRT10, KRT6A, KRT9, MB, MYOM1, PCDHB12, PRNP, SERPINA3 \\
Diabetes & DPP4, REG1A, REG1B \\
\hline
\end{tabular}

Human plasma has innumerable links with other body fluids, which has a potentially important impact on the human body. Taking this into consideration, we proposed a model illustrating the possible connection of plasma with these body fluids, which provided an additional layer for a deep understanding of the human body. We supposed that KJF may enter the plasma through an unknown mechanism due to $87 \%$ overlap between KJF and plasma, implying the potential application of plasma biomarkers in KJF. Additionally, combined with previous studies related to multiple sclerosis, we also proposed a possible clue between CSF and plasma. The focus of many research works has been to expand the pool of available biomarkers in precision medicine and personalized nutrition for many years. The integrated proteomic profiling of ten body fluids provided an overall cognition and understanding of the associations and differences among these fluids. Identification of specificity and commonality of these samples at the molecular level may also facilitate with biomarker discovery and their use in the clinical setting.

The present study provides a rich resource for the proteome of human body fluids. The development of new biomarkers may improve the accuracy of diagnosis and therapy of many diseases, including cancer; thus, allowing clinical oncology to enter the area of precision medicine. CSF is a source of brain tumor biomarkers. Brain tumor can cause hemorrhage; it has been reported that the incidence of hemorrhage from pituitary adenoma is higher than other brain tumors (49). In this study, we included one SAH patient and identified 116 new proteins in CSF that have not been reported in previous studies. The differences between proteomes of SAH and brain tumor with hemorrhage may increase the accuracy of CSF biomarkers for brain tumor diagnosis. Further larger studies are needed to establish a proteome baseline of CSF.

In addition, urine is also a desirable material for the diagnosis and classification of diseases and can prove useful in biomarker discovery. As shown in Table $\mathrm{V}$, we searched our urinary proteome data for common disease-associated urine markers and found 23 markers associated with arthritis. These included ACAN, ADAMTS1, ADAMTS2, ANXA5, BSG, CD5L, CILP, COMP, CRP, CSTA, FLG, GPI, HLA-DRB1, IL1RN, ITIH1, ITIH3, LCN2, MMP9, ORM2, PRTN3, S100A8, S100A9 and TIMP1. Besides that, we also identified 13 markers associated with myositis, such as CANX, DES, HLA-A, HLA-DRB1, ICAM1, KRT10, KRT6A, KRT9, MB, MYOM1, PCDHB12, PRNP and SERPINA3. In our study, diabetes-related urine markers DPP4, REG1A and REG1B were also detected.

In conclusion, a comprehensive proteome draft of ten human body fluids, including ascites, bile, CSF, hydrothorax, KJF, plasma, saliva, serum, tear fluid, and urine, was obtained using MS-based shotgun proteomics. The proteome profiling revealed the common functions in focal adhesion and complement/ coagulation cascades, and body-specific protein and function features, and the unknown associations among body fluids. The result provides an original shared data resource, contributing to the understanding of human body fluids.

\section{Conflicts of Interest}

The Authors have no conflicts of interest to declare.

\section{Authors' Contributions}

Conceptualization, Li Y. and Ding C.; Investigation, Xun D., Li L. and Wang B.; Resources, Lv J., Liu H. and Chen X.; Data Curation, Xun D., Lv J., Zhu L., Ma F., and Tian S.; Writing, Li Y. and Ding C.; Funding Acquisition, Ding C.

\section{Acknowledgements}

This work is supported by the Chinese Ministry of Science and Technology (2016YFA0502500), National International Cooperation Grant (2014DFB30010), National Program on Key Basic Research Project (973 Program, 2012CB910300, 2014CBA02000), National High-tech R\&D Program of China (863 program, 2014AA020201), National Natural Science Foundation of China (31270822) and Beijing Natural Science Foundation (Z131100005213003). We thank Dr. Shen Nan from Shanghai Children's Medical Center, Shanghai Jiao Tong University School of Medicine for revising the manuscript.

\section{References}

1 Peffers MJ, McDermott B, Clegg PD and Riggs CM: Comprehensive protein profiling of synovial fluid in osteoarthritis following protein equalization. Osteoarthritis Cartilage 23(7): 12041213, 2015. PMID: 25819577. DOI: 10.1016/j.joca.2015.03.019 
2 Aebersold R and Mann M: Mass spectrometry-based proteomics. Nature 422(6928): 198-207, 2003. PMID: 12634793. DOI: 10.1038 /nature 01511

3 Yates JR 3rd: Mass spectral analysis in proteomics. Annu Rev Biophys Biomol Struct 33: 297-316, 2004. PMID: 15139815. DOI: 10.1146/annurev.biophys.33.111502.082538

4 Veenstra TD, Conrads TP, Hood BL, Avellino AM, Ellenbogen RG and Morrison RS: Biomarkers: mining the biofluid proteome. Mol Cell Proteomics 4(4): 409-418, 2005. PMID: 15684407. DOI: $10.1074 / \mathrm{mcp} . M 500006-\mathrm{MCP} 200$

5 Schulze WX and Usadel B: Quantitation in mass-spectrometrybased proteomics. Annu Rev Plant Biol 61: 491-516, 2010. PMID: 20192741. DOI: 10.1146/annurev-arplant-042809-112132

6 Gygi SP, Corthals GL, Zhang Y, Rochon Y and Aebersold R: Evaluation of two-dimensional gel electrophoresis-based proteome analysis technology. Proc Natl Acad Sci USA 97(17): 9390-9395, 2000. PMID: 10920198. DOI: 10.1073/pnas.160270797

7 Margolis J and Kenrick KG: 2-dimensional resolution of plasma proteins by combination of polyacrylamide disc and gradient gel electrophoresis. Nature 221(5185): 1056-1057, 1969. PMID: 5774398. DOI: $10.1038 / 2211056 \mathrm{a0}$

8 Tang J, Gao M, Deng C and Zhang X: Recent development of multi-dimensional chromatography strategies in proteome research. J Chromatogr B Analyt Technol Biomed Life Sci 866(1-2): 123-132, 2008. PMID: 18289947. DOI: 10.1016/ j.jchromb.2008.01.029

9 Zhao YY and Lin RC: UPLC-MS(E) application in disease biomarker discovery: the discoveries in proteomics to metabolomics. Chem Biol Interact 215: 7-16, 2014. PMID: 24631021. DOI: 10.1016/j.cbi.2014.02.014

10 Unlü M, Morgan ME and Minden JS: Difference gel electrophoresis: a single gel method for detecting changes in protein extracts. Electrophoresis 18(11): 2071-2077, 1997. PMID: 9420172. DOI: 10.1002/elps.1150181133

11 Gygi SP, Rist B, Gerber SA, Turecek F, Gelb MH and Aebersold R: Quantitative analysis of complex protein mixtures using isotope-coded affinity tags. Nat Biotechnol 17(10): 994-999. 1999. PMID: 10504701 . DOI: $10.1038 / 13690$

12 Hoedt E, Zhang G and Neubert TA: Stable isotope labeling by amino acids in cell culture (SILAC) for quantitative proteomics. Adv Exp Med Biol 806: 93-106, 2014. PMID: 24952180. DOI: 10.1007/978-3-319-06068-2_5

13 Hoedt E, Zhang G and Neubert TA: Stable isotope labeling by amino acids in cell culture (SILAC) for quantitative proteomics. Adv Exp Med Biol 1140: 531-539, 2019. PMID: 31347069. DOI: 10.1007/978-3-030-15950-4_31

14 Ross PL, Huang YN, Marchese JN, Williamson B, Parker K, Hattan S, Khainovski N, Pillai S, Dey S, Daniels S, Purkayastha S, Juhasz P, Martin S, Bartlet-Jones M, He F, Jacobson A and Pappin DJ: Multiplexed protein quantitation in Saccharomyces cerevisiae using amine-reactive isobaric tagging reagents. Mol Cell Proteomics 3(12): 1154-1169, 2004. PMID: 15385600. DOI: $10.1074 / \mathrm{mcp} . M 400129-\mathrm{MCP} 200$

15 De Bock M, de Seny D, Meuwis MA, Chapelle JP, Louis E, Malaise M, Merville MP and Fillet M: Challenges for biomarker discovery in body fluids using SELDI-TOF-MS. J Biomed Biotechnol 2010: 906082, 2010. PMID: 20029632. DOI: $10.1155 / 2010 / 906082$

16 Tolstikov VV, Lommen A, Nakanishi K, Tanaka N and Fiehn O: Monolithic silica-based capillary reversed-phase liquid chromatography/electrospray mass spectrometry for plant metabolomics. Anal Chem 75(23): 6737-6740, 2003. PMID: 14640754. DOI: $10.1021 / \mathrm{ac} 034716 \mathrm{z}$

17 Vitorino R, Guedes S, Manadas B, Ferreira R and Amado F: Toward a standardized saliva proteome analysis methodology. J Proteomics 75(17): 5140-5165, 2012. PMID: 22809520. DOI: 10.1016/j.jprot.2012.05.045

18 Bantscheff M, Schirle M, Sweetman G, Rick J and Kuster B: Quantitative mass spectrometry in proteomics: a critical review. Anal Bioanal Chem 389(4): 1017-1031, 2007. PMID: 17668192. DOI: $10.1007 / \mathrm{s} 00216-007-1486-6$

19 Neilson KA, Ali NA, Muralidharan S, Mirzaei M, Mariani M, Assadourian G, Lee A, van Sluyter SC and Haynes PA: Less label, more free: approaches in label-free quantitative mass spectrometry. Proteomics 11(4): 535-553, 2011. PMID: 21243637. DOI: $10.1002 /$ pmic. 201000553

20 Omenn GS: The Human Proteome Organization Plasma Proteome Project pilot phase: reference specimens, technology platform comparisons, and standardized data submissions and analyses. Proteomics 4(5): 1235-1240, 2004. PMID: 15188391. DOI: $10.1002 /$ pmic. 200300686

21 Schmidt A and Aebersold R: High-accuracy proteome maps of human body fluids. Genome Biol 7(11): 242, 2006. PMID: 17140426. DOI: $10.1186 / \mathrm{gb}-2006-7-11-242$

22 Adachi J, Kumar C, Zhang Y, Olsen JV and Mann M: The human urinary proteome contains more than 1500 proteins, including a large proportion of membrane proteins. Genome Biol 7(9): R80, 2006. PMID: 16948836 . DOI: 10.1186/gb-2006-7-9-R80

23 Pilch B and Mann M: Large-scale and high-confidence proteomic analysis of human seminal plasma. Genome Biol 7(5): R40, 2006. PMID: 16709260. DOI: 10.1186/gb-2006-7-5-r40

24 Wilhelm M, Schlegl J, Hahne H, Gholami AM, Lieberenz M, Savitski MM, Ziegler E, Butzmann L, Gessulat S, Marx H, Mathieson T, Lemeer S, Schnatbaum K, Reimer U, Wenschuh H, Mollenhauer M, Slotta-Huspenina J, Boese JH, Bantscheff M, Gerstmair A, Faerber F and Kuster B: Mass-spectrometry-based draft of the human proteome. Nature 509(7502): 582-587, 2014. PMID: 24870543. DOI: 10.1038/nature13319

25 Goodison S, Rosser CJ and Urquidi V: Urinary proteomic profiling for diagnostic bladder cancer biomarkers. Expert Rev Proteomics 6(5): 507-514, 2009. PMID: 19811072. DOI: 10.1586/epr.09.70

26 Li C, Zang T, Wrobel K, Huang JT and Nabi G: Quantitative urinary proteomics using stable isotope labelling by peptide dimethylation in patients with prostate cancer. Anal Bioanal Chem 407(12): 3393-3404, 2015. PMID: 25724369. DOI: 10.1007/s00216-015-8569-6

$27 \varnothing$ vrehus MA, Zürbig P, Vikse BE and Hallan SI: Urinary proteomics in chronic kidney disease: diagnosis and risk of progression beyond albuminuria. Clin Proteomics 12(1): 21, 2015. PMID: 26257595. DOI: 10.1186/s12014-015-9092-7

28 Ditzen C, Tang N, Jastorff AM, Teplytska L, Yassouridis A, Maccarrone G, Uhr M, Bronisch T, Miller CA, Holsboer F and Turck CW: Cerebrospinal fluid biomarkers for major depression confirm relevance of associated pathophysiology. Neuropsychopharmacology 37(4): 1013-1025, 2012. PMID: 22169944. DOI: $10.1038 / n p p .2011 .285$

29 Stoop MP, Coulier L, Rosenling T, Shi S, Smolinska AM, Buydens L, Ampt K, Stingl C, Dane A, Muilwijk B, Luitwieler RL, Sillevis Smitt PA, Hintzen RQ, Bischoff R, Wijmenga SS, Hankemeier T, van Gool AJ and Luider TM: Quantitative 
proteomics and metabolomics analysis of normal human cerebrospinal fluid samples. Mol Cell Proteomics 9(9): 20632075, 2010. PMID: 20811074. DOI: 10.1074/mcp.M900877MCP200

30 Ramachandran P, Boontheung P, Xie Y, Sondej M, Wong DT and Loo JA: Identification of N-linked glycoproteins in human saliva by glycoprotein capture and mass spectrometry. J Proteome Res 5(6): 1493-1503, 2006. PMID: 16740002. DOI: 10.1021/pr050492k

31 Schwanhäusser B, Busse D, Li N, Dittmar G, Schuchhardt J, Wolf J, Chen W and Selbach M: Global quantification of mammalian gene expression control. Nature 473(7347): $337-$ 342, 2011. PMID: 21593866. DOI: 10.1038/nature 10098

32 Tyanova S, Temu T and Cox J: The MaxQuant computational platform for mass spectrometry-based shotgun proteomics. Nat Protoc 11(12): 2301-2319, 2016. PMID: 27809316. DOI: 10.1038/nprot.2016.136

33 Lambert I, Paysant-Le Roux C, Colella S and Martin-Magniette ML: DiCoExpress: a tool to process multifactorial RNAseq experiments from quality controls to co-expression analysis through differential analysis based on contrasts inside GLM models. Plant Methods 16: 68, 2020. PMID: 32426025. DOI: 10.1186/s13007-020-00611-7

34 Wilkerson MD and Hayes DN: ConsensusClusterPlus: a class discovery tool with confidence assessments and item tracking. Bioinformatics 26(12): 1572-1573, 2010. PMID: 20427518. DOI: $10.1093 /$ bioinformatics/btq170

35 Marouga R, David S and Hawkins E: The development of the DIGE system: 2D fluorescence difference gel analysis technology. Anal Bioanal Chem 382(3): 669-678, 2005. PMID: 15900442. DOI: 10.1007/s00216-005-3126-3

36 Ma J, Chen T, Wu S, Yang C, Bai M, Shu K, Li K, Zhang G, Jin $\mathrm{Z}, \mathrm{He}$ F, Hermjakob $\mathrm{H}$ and Zhu Y: iProX: an integrated proteome resource. Nucleic Acids Res 47(D1): D1211-D1217, 2019. PMID: 30252093. DOI: 10.1093/nar/gky869

37 Yan W, Apweiler R, Balgley BM, Boontheung P, Bundy JL, Cargile BJ, Cole S, Fang X, Gonzalez-Begne M, Griffin TJ, Hagen F, Hu S, Wolinsky LE, Lee CS, Malamud D, Melvin JE, Menon R, Mueller M, Qiao R, Rhodus NL, Sevinsky JR, States D, Stephenson JL, Than S, Yates JR, Yu W, Xie H, Xie Y, Omenn GS, Loo JA and Wong DT: Systematic comparison of the human saliva and plasma proteomes. Proteomics Clin Appl 3(1): 116134, 2009. PMID: 19898684. DOI: 10.1002/prca.200800140

38 Muthusamy B, Hanumanthu G, Suresh S, Rekha B, Srinivas D, Karthick L, Vrushabendra BM, Sharma S, Mishra G, Chatterjee P, Mangala KS, Shivashankar HN, Chandrika KN, Deshpande N, Suresh M, Kannabiran N, Niranjan V, Nalli A, Prasad TS, Arun KS, Reddy R, Chandran S, Jadhav T, Julie D, Mahesh M, John SL, Palvankar K, Sudhir D, Bala P, Rashmi NS, Vishnupriya G, Dhar K, Reshma S, Chaerkady R, Gandhi TK, Harsha HC, Mohan SS, Deshpande KS, Sarker M and Pandey A: Plasma Proteome Database as a resource for proteomics research. Proteomics 5(13): 3531-3536, 2005. PMID: 16041672. DOI: $10.1002 /$ pmic.200401335

39 Nanjappa V, Thomas JK, Marimuthu A, Muthusamy B, Radhakrishnan A, Sharma R, Ahmad Khan A, Balakrishnan L, Sahasrabuddhe NA, Kumar S, Jhaveri BN, Sheth KV, Kumar Khatana R, Shaw PG, Srikanth SM, Mathur PP, Shankar S,
Nagaraja D, Christopher R, Mathivanan S, Raju R, Sirdeshmukh R, Chatterjee A, Simpson RJ, Harsha HC, Pandey A and Prasad TS: Plasma Proteome Database as a resource for proteomics research: 2014 update. Nucleic Acids Res 42(Database issue): D959-D965, 2014. PMID: 24304897. DOI: 10.1093/nar/gkt1251

40 Zhang Y, Guo Z, Zou L, Yang Y, Zhang L, Ji N, Shao C, Sun W and Wang Y: A comprehensive map and functional annotation of the normal human cerebrospinal fluid proteome. J Proteomics 119: 90-99, 2015. PMID: 25661039. DOI: 10.1016/j.jprot.2015.01.017

41 Kentsis A, Monigatti F, Dorff K, Campagne F, Bachur R and Steen $\mathrm{H}$ : Urine proteomics for profiling of human disease using high accuracy mass spectrometry. Proteomics Clin Appl 3(9): 10521061, 2009. PMID: 21127740. DOI: 10.1002/prca.200900008

42 Lin YH, Eguez RV, Torralba MG, Singh H, Golusinski P, Golusinski W, Masternak M, Nelson KE, Freire M and Yu Y: Self-assembled STrap for global proteomics and salivary biomarker discovery. J Proteome Res 18(4): 1907-1915, 2019. PMID: 30848925. DOI: 10.1021/acs.jproteome.9b00037

43 Jin J, Son M, Kim H, Kim H, Kong SH, Kim HK, Kim Y and Han D: Comparative proteomic analysis of human malignant ascitic fluids for the development of gastric cancer biomarkers. Clin Biochem 56: 55-61, 2018. PMID: 29654727. DOI: 10.1016/j.clinbiochem.2018.04.003

44 Farina A, Delhaye M, Lescuyer P and Dumonceau JM: Bile proteome in health and disease. Compr Physiol 4(1): 91-108, 2014. PMID: 24692135. DOI: 10.1002/cphy.c130016

45 Macron C, Lane L, Núñez Galindo A and Dayon L: Deep dive on the proteome of human cerebrospinal fluid: A valuable data resource for biomarker discovery and missing protein identification. J Proteome Res 17(12): 4113-4126, 2018. PMID: 30124047. DOI: 10.1021/acs.jproteome.8b00300

46 Bhattacharjee M, Balakrishnan L, Renuse S, Advani J, Goel R, Sathe G, Keshava Prasad TS, Nair B, Jois R, Shankar S and Pandey A: Synovial fluid proteome in rheumatoid arthritis. Clin Proteomics 13: 12, 2016. PMID: 27274716. DOI: 10.1186/ s12014-016-9113-1

47 Ai J, Smith B and Wong DT: Saliva Ontology: an ontologybased framework for a Salivaomics Knowledge Base. BMC Bioinformatics 11: 302, 2010. PMID: 20525291. DOI: 10.1186/ 1471-2105-11-302

48 de Souza GA, Godoy LM and Mann M: Identification of 491 proteins in the tear fluid proteome reveals a large number of proteases and protease inhibitors. Genome Biol 7(8): R72, 2006. PMID: 16901338. DOI: 10.1186/gb-2006-7-8-R72

49 Wakai S, Yamakawa K, Manaka S and Takakura K: Spontaneous intracranial hemorrhage caused by brain tumor: its incidence and clinical significance. Neurosurgery 10(4): 437-444, 1982. PMID: 7099393. DOI: $10.1227 / 00006123-198204000-00004$
Received March 8, 2021

Revised June 2, 2021

Accepted June 3, 2021 